\title{
Composición, diversidad y estructura de especies leñosas en los bosques templados de Monte Grande, Sierra Fría, Aguascalientes, México
}

\author{
Composition, diversity and structure of woody species \\ in temperate forests of Monte Grande, Sierra Fría, \\ Aguascalientes, Mexico
}

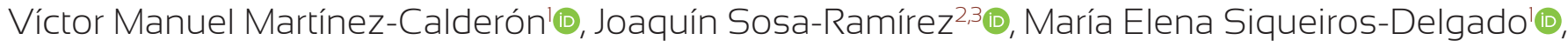 \\ Vicente Díaz-Núñez² (1)
}

\section{Resumen}

Antecedentes y Objetivos: Dentro del Área Natural Protegida Sierra Fría (ANPSF), Aguascalientes, México, se han llevado a cabo algunos estudios sobre la composición, diversidad y dinámica de los bosques templados en zonas bajas; en este trabajo se estudia Monte Grande, su zona más alta. El objetivo de esta investigación fue caracterizar la composición, la diversidad y la estructura de las comunidades de especies leñosas en los bosques templados de Monte Grande, dentro del ANPSF.

Métodos: Los individuos con un diámetro basal $\geq 5 \mathrm{~cm}$ se midieron en 32 transectos de $300 \mathrm{~m}^{2}$. Se realizó una agrupación por medio de un análisis cluster y posteriormente los datos se analizaron mediante pruebas de Kruskal-Wallis y ANOVA para encontrar diferencias entre asociaciones vegetales. Los datos analizados incluyeron densidad, área basal, altura, valor de importancia relativa, riqueza y número de especies efectivas.

Resultados clave: Veintiséis especies leñosas (nueve géneros y ocho familias) se identificaron en cinco asociaciones: bosque de encino de Quercus sideroxyla-Quercus rugosa, bosque de encino de Quercus potosina-Arctostaphylos pungens, bosque mixto de Quercus-Pinus, bosque mixto de Juniperus-Quercus y bosque de Juniperus. El bosque de $Q$. sideroxyla-Q. rugosa presentó la mayor área basal (53 $\mathrm{m}^{2} / \mathrm{ha}$ ) y el bosque de QuercusPinus, la mayor densidad (2200 ind/ha). El número de especies efectivas oscila entre $1.50-4.03^{1} D$ y $1.27-3.24^{2} D$. La abundancia de $A$. pungens y $J$. deppeana, consideradas pioneras, fue alta.

Conclusiones: La estructura y diversidad de las asociaciones presentaron diferencias debidas a su composición, condiciones en las que se desarrollan e historial de manejo de Monte Grande. La información generada proporciona un marco de referencia para la toma de decisiones en relación con la conservación y aprovechamiento de los bosques de la región.

Palabras clave: bosque de encino, bosque mixto, composición florística, conservación, especies efectivas, Sierra Madre Occidental.

\section{Abstract:}

Background and Aims: Within the Natural Protected Area Sierra Fría (ANPSF), Aguascalientes, Mexico, some studies have been carried out on the composition, diversity and dynamics of temperate forests in lowlands; in this work we studied Monte Grande, its highest area. The aim of this study was to characterize the composition, diversity and structure of the woody species communities in the temperate forests of Monte Grande, within the ANPSF.

Methods: Individuals with a basal diameter $\geq 5 \mathrm{~cm}$ were measured in 32 transects of $300 \mathrm{~m}^{2}$. A grouping by cluster analysis and subsequently KruskalWallis and ANOVA tests were performed to find differences between plant associations. The analyzed data included density, basal area, height, relative importance value, richness and effective number of species.

Key results: Twenty-six woody species (nine genera and eight families) were identified in five associations: Quercus sideroxyla-Quercus rugosa oak forest, Quercus potosina-Arctostaphylos pungens oak forest, Quercus-Pinus mixed forest, Juniperus-Quercus mixed forest and Juniperus forest. The Q. sideroxyla-Q. rugosa forest had the largest basal area value $\left(53 \mathrm{~m}^{2} / \mathrm{ha}\right)$, and the Quercus-Pinus forest had the highest density ( 2200 ind/ha). The effective number of species varied between $1.50-4.03^{1} D$ 1.27-3.24 $D$. The abundance of $A$. pungens and J. deppeana, considered pioneers, was high. Conclusions: The structure and diversity of the associations presented differences due to their composition, the conditions in which the associations develop, and the management history of Monte Grande. The generated information provides a reference frame for decision making in relation to the conservation and use of the forests of the region.

Key words: conservation, effective species, floristic composition, mixed forest, oak forest, Sierra Madre Occidental.

\author{
${ }^{1}$ Universidad Autónoma de Aguascalientes, Cen- \\ tro de Ciencias Básicas, Av. Universidad 940, Ciudad \\ Universitaria, 20100 Aguascalientes, Aguascalientes, \\ México. \\ 2Universidad Autónoma de Aguascalientes, Centro de \\ Ciencias Agropecuarias, Av. Universidad 940, Ciudad \\ Universitaria, 20100 Aguascalientes, Aguascalientes, \\ México. \\ ${ }^{3}$ Autor de correspondencia: jsosar@correo.uaa.mx
}

Recibido: 4 de diciembre de 2020

Revisado: 5 de febrero de 2021.

Aceptado por Moisés Méndez Toribio: 3 de agosto de 2021.

Publicado Primero en línea: 12 de agosto de 2021.

Publicado: Acta Botanica Mexicana 128(2021).

(c) (1) Este es un artículo de acceso abierto Br $\mathbf{N C}$ bajo la licencia Creative Commons 4.0 Atribución-No Comercial (CC BY-NC 4.0 Internacional).
Citar como: Martínez-Calderón, V. M., J. SosaRamírez, M. E. Siqueiros-Delgado y V. Díaz-Núñez. 2021. Composición, diversidad y estructura de especies leñosas en los bosques templados de Monte Grande, Sierra Fría, Aguascalientes, México. Acta Botanica Mexicana 128: e1829. DOI: https://doi.org/10.21829/abm128.2021.1829

e-ISSN: $2448-7589$ 


\section{Introducción}

Los bosques templados son comunidades dominadas principalmente por especies de coníferas y Quercus L., presentes generalmente en climas templados y fríos (Rzedowski, 2006). En México se encuentran, en su mayoría, en los sistemas montañosos del país, así como en serranías y montañas aisladas en el Altiplano y además entremezclados en las planicies tropicales (Challenger y Soberón, 2008); albergan especies de gran relevancia para la investigación científica, de alta importancia por los servicios ecosistémicos que proveen y de alto interés para la economía nacional (Sánchez et al., 2003).

Similar a lo que ocurre en el mundo, en México se ha observado una disminución en la superficie boscosa; de 1976 a 2007 se perdieron 8806 km² de bosque templado (Rosete-Vergés et al., 2014). Esa pérdida amenaza su conservación, pues son vulnerables a disturbios antrópicos como la extracción de madera, la ganadería y los incendios forestales (Challenger, 2003; Rzedowski, 2006), lo que llega a ocasionar cambios en la estructura de los bosques y genera comunidades en distintas fases de sucesión (Álvarez-Yépiz et al., 2008; Calderón-Aguilera et al., 2012; Almazán-Núñez et al., 2016). Esto hace necesario generar información cuantitativa en los bosques templados, ya que además de su amplia distribución nacional, son la base de la industria maderera en México (Sáenz-Romero et al., 2003; Zacarias-Eslava et al., 2011). Cabe destacar que, a diferencia de los bosques tropicales y mesófilos de montaña, los bosques templados han sido poco estudiados, lo que provoca que la información disponible de las zonas templadas sea comparativamente escasa (Zacarias-Eslava et al., 2011).

La gestión forestal requiere de datos acerca de la estructura, crecimiento, producción y regeneración natural de los bosques para detectar cambios en su estado (Aguirre et al., 2008). Las características estructurales de la comunidad son un buen indicador de la biodiversidad del sistema y es fácilmente modificable a través de prácticas de silvicultura, por lo que su adecuado conocimiento es indispensable para garantizar una gestión sostenible (Del Río et al., 2003). Por eso, los estudios sobre la composición, estructura y diversidad de la vegetación aportan datos importantes para establecer estrategias y lograr un uso sustentable de los recursos forestales (López-Hernández et al., 2017). Esta infor- mación apoya la toma de decisiones en el manejo de áreas boscosas donde se observan procesos de sucesión natural y efectos antropogénicos (Zacarias-Eslava et al., 2011; LópezHernández et al., 2017).

En el estado de Aguascalientes la vegetación de clima templado cubre aproximadamente $30.48 \%$ de su superficie total; los bosques mixtos y de Quercus son los más abundantes y se distribuyen principalmente en el Área Natural Protegida Sierra Fría (ANPSF) (Siqueiros et al., 2016). Los disturbios antrópicos ocurridos a principios del Siglo XX en esta ANP provocaron, sobre todo en las zonas accesibles, una fragmentación del bosque (Minnich et al., 1994; ChapaBezanilla et al., 2008). Sin embargo, a finales de la década de 1950, el ANPSF inició un proceso de regeneración (Minnich et al., 1994). Las especies que mayormente han colonizado los sitios que han sido impactados con actividades humanas son Juniperus deppeana Steud. y Arctostaphylos pungens Kunth (Díaz-Núñez et al., 2016).

En el ANPSF se han realizado varios estudios, los cuales van desde el análisis de la distribución de especies leñosas y los factores que la determinan hasta el reconocimiento de la dinámica temporal de los paisajes (Chapa-Bezanilla et al., 2008; Díaz-Núñez et al., 2012, 2016). Sin embargo, existen sitios dentro del ANP que no han sido estudiados y la estructura de su vegetación es aún desconocida; es el caso del bosque templado en la propiedad comunal "Monte Grande". Este es un sitio de difícil acceso dentro del ANPSF que no cuenta con información sobre la estructura de su vegetación; ni ha sido tomado en cuenta, gran parte de su extensión, para realizar estudios a pesar de ser considerado una zona importante en la conservación de los recursos en Aguascalientes (CONABIO, 2008). Monte Grande, al ser el sitio de mayor altitud (Siqueiros et al., 2016), presenta condiciones climáticas distintas en temperatura y precipitación, lo que debería derivar en que la composición y la estructura difieran en comparación con las zonas de menor altitud dentro del ANP. Lo anterior hace necesario realizar estudios en la zona para lograr un mayor entendimiento de los bosques templados de Aguascalientes, dado el desconocimiento que aun impera en los bosques templados del país.

Los estudios sobre la estructura de los bosques templados en el ANPSF son importantes por la función que 
cumplen en ella. Los bosques templados del sitio de estudio y el resto del ANP fungen como proveedores de beneficios económicos y brindan servicios ecosistémicos, especialmente hidrológicos, pues el ANP tiene una disponibilidad hídrica estimada de 51.3 millones de metros cúbicos y está rodeada de zonas semiáridas y agrícolas que dependen de la captación de agua por parte de los bosques del ANPSF (COTAS, 2006; Martín-Clemente et al., 2015; Sosa-Ramírez et al., 2015). Estos beneficios hidrológicos son compartidos mayormente en los estados de Aguascalientes y Zacatecas (COTAS, 2006).

Por lo anterior, el objetivo del presente estudio consistió en caracterizar la composición, la diversidad y la estructura de las comunidades de especies leñosas en los bosques templados de Monte Grande dentro del ANPSF, y de esta manera generar información base para futuras investigaciones y apoyar la correcta toma de decisiones para el manejo y conservación de los recursos forestales. La hipótesis de trabajo sugiere que predominarán las especies de sucesión secundaria en la comunidad debido a la influencia del manejo que se ha llevado a cabo en Monte Grande en décadas anteriores.

\section{Materiales y Métodos}

\section{Descripción del área de estudio}

El estudio se realizó en los bosques de la propiedad comunal Monte Grande, Aguascalientes, México. El área cuenta con una superficie de 3852 ha, está ubicada en la parte noroeste del ANPSF entre las coordenadas $102^{\circ} 38^{\prime} 22^{\prime \prime}$ a $102^{\circ} 33^{\prime} 12^{\prime \prime}$ longitud oeste y $22^{\circ} 17^{\prime} 01^{\prime \prime}$ a $22^{\circ} 13^{\prime} 27^{\prime \prime}$ latitud norte (Fig. 1). Presenta una altitud de 2480 a 3030 m, siendo la zona de mayor altitud en el estado. En el área de estudio la vegetación está dominada por bosques templados de Quercus, Pinus-Quercus, Juniperus-Quercus, así como por matorral templado y algunas zonas de pastizal templado, siendo una de las propiedades mejor conservadas en el ANPSF (CONABIO, 2008). El clima es de tipo templado subhúmedo con lluvias en verano, con una temperatura media anual de $12-18{ }^{\circ} \mathrm{C}$ y precipitación media anual de $600-800$ $\mathrm{mm}$; el ANPSF pertenece a la región hidrológica LermaSantiago (INEGI, 2000; CONAFOR, 2014). Los suelos en su mayoría son someros de tipo luvisol y se presentan rocas de tipo ígnea extrusiva (CONABIO, 2008).
Monte Grande es la propiedad comunal con mayor extensión dentro del ANPSF, en la cual aún se encuentran sitios conservados en zonas de difícil acceso (Minnich et al., 1994). En la actualidad, el manejo de la propiedad está enfocado a la protección de la biodiversidad y los servicios ecosistémicos. Las actividades de aprovechamiento concluyeron desde hace aproximadamente cuatro décadas y el acceso a propiedades colindantes de Aguascalientes y Zacatecas fueron cerrados (L. Suarez, com. pers., 2018).

\section{Diseño de muestreo}

Se elaboró un plan de muestreo estratificado (Daget y Godron, 1982), con el fin de cubrir las distintas asociaciones boscosas que pudieran encontrarse en el área de estudio. Los estratos de muestreo se delimitaron con base en la altitud, geoforma del sitio (terrenos planos, cóncavos y convexos) y exposición solar (N, S, E, O). La geoforma se obtuvo con base en la pendiente, donde terrenos planos=sitios con pendiente $\leq 10 \%$, terrenos cóncavos=baja pendiente $y$ depresión ( $>10 \%$ y $<25 \%$ ), y terrenos convexos=alta y media pendiente ( $>25 \%)$. La altitud se dividió en tres estratos cada 200 m: I) <2600, II) 2600-2800, III) >2800, siendo los últimos dos estratos los que presentan la mayor extensión.

Se analizaron 32 sitios conforme al diseño de muestreo (Fig. 1, Cuadro 1) durante 2017 y 2018. En cada sitio se estableció una parcela de $300 \mathrm{~m}^{2}(50 \times 6 \mathrm{~m})$ con el lado mayor de manera perpendicular a la pendiente para facilitar el trabajo en sitios de alta inclinación. En cada una de las parcelas se contaron y midieron todas las especies arbóreas y arbustivas con un diámetro $\geq 5 \mathrm{~cm}$ (Olvera-Vargas y Figueroa-Rangel, 2012). El diámetro fue medido a $20 \mathrm{~cm}$ $\left(\mathrm{d}_{0.20}\right)$ sobre la base del suelo, debido a que las especies presentaron múltiples bifurcaciones a mayor altura y desde la base del fuste (Alanís-Rodríguez et al., 2012); en especial especies como Arctostaphylos pungens y Juniperus deppeana, así como especies de Quercus que llegan a presentar múltiples bifurcaciones desde la base, lo que hace recomendable medir el diámetro a la base para conocer el área basal (Mueller-Dombois y Ellenberg, 1974). Cuando el árbol presentó dos o más bifurcaciones a partir de la base, se midieron el diámetro de cada fuste y la altura del fuste principal. Cuando el árbol presentó una o más bifurcaciones a partir de una altura $\geq 1.50 \mathrm{~m}$, se midió únicamente el 


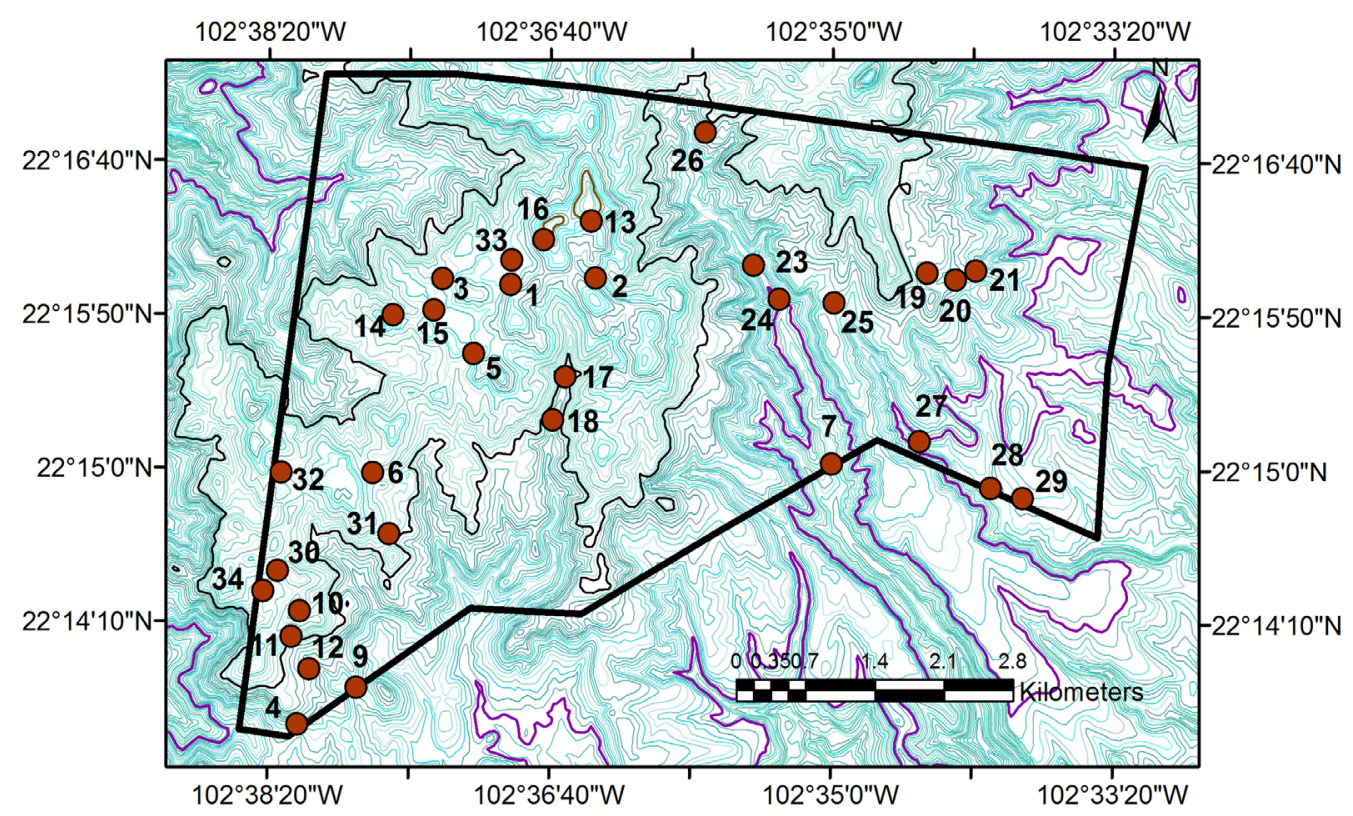

O Sitios de muestreo

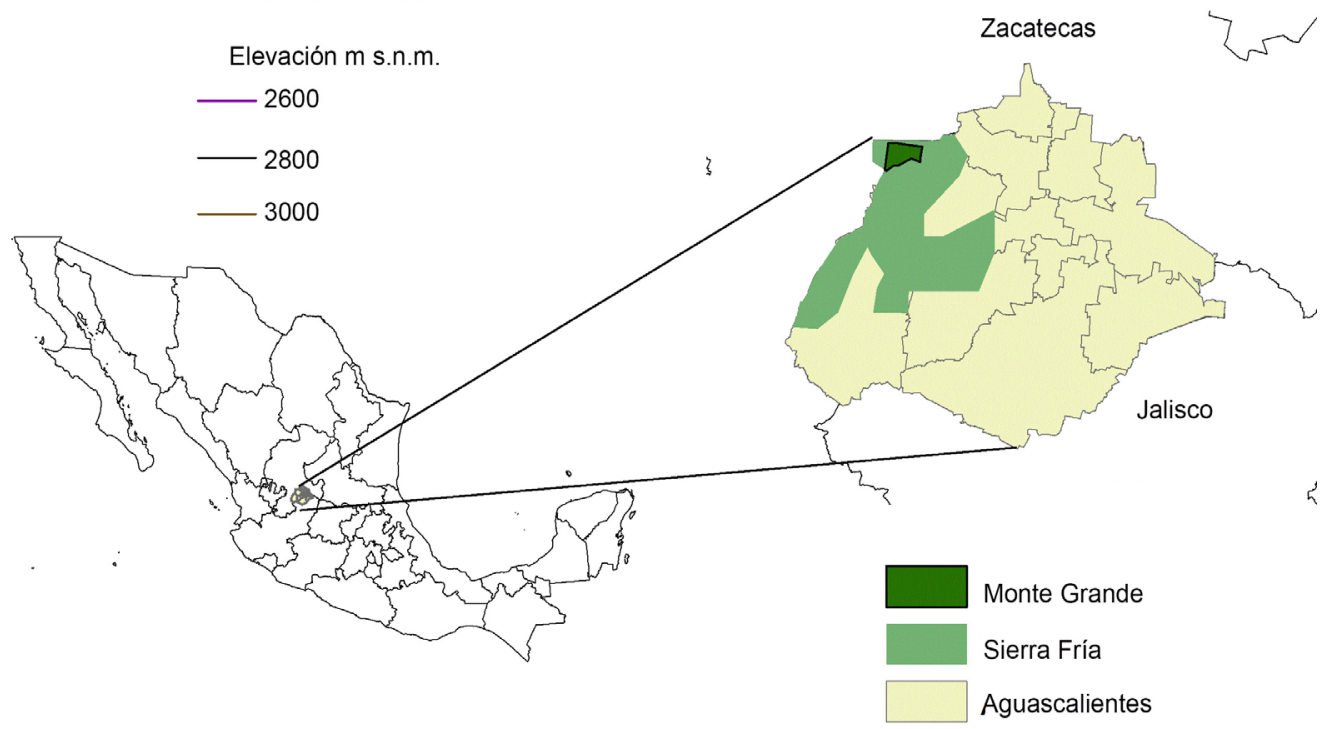

Figura 1: Ubicación de la localidad de Monte Grande, dentro del polígono del Área Natural Protegida Sierra Fría, Aguascalientes, México.

Cuadro 1: Matriz de sitios de muestreo en niveles altitudinales, geoforma del sitio y exposición solar de la ladera derivados del sistema de muestreo en Monte Grande, dentro del Área Natural Protegida Sierra Fría, Aguascalientes, México. *Plano=sitios con pendiente $\leq 10 \%$; Cóncava=baja pendiente y depresión (>10\% y <25\%); Convexa=alta y media pendiente ( $>25 \%)$.

\section{Geoforma del sitio}

\begin{tabular}{|c|c|c|c|c|c|c|c|c|c|c|}
\hline \multirow{2}{*}{$\begin{array}{l}\text { Niveles } \\
\text { altitudinales }\end{array}$} & \multicolumn{4}{|c|}{ Cóncava } & \multicolumn{4}{|c|}{ Convexa } & \multirow[t]{2}{*}{ Plano } & \multirow[t]{2}{*}{ Total } \\
\hline & $\mathbf{N}$ & $S$ & E & 0 & $\mathbf{N}$ & $S$ & E & 0 & & \\
\hline$<2600 m$ & 0 & 1 & 0 & 1 & 0 & 0 & 0 & 0 & 3 & 5 \\
\hline $2600-2800 \mathrm{~m}$ & 1 & 2 & 1 & 2 & 1 & 2 & 1 & 1 & 2 & 13 \\
\hline$>2800 \mathrm{~m}$ & 1 & 0 & 0 & 1 & 3 & 2 & 1 & 2 & 4 & 14 \\
\hline Total & 2 & 3 & 1 & 4 & 4 & 4 & 2 & 3 & 9 & 32 \\
\hline
\end{tabular}


fuste principal. Se tomó la altura total con ayuda de un altímetro (Haga GmbH + Co KG, Alemania), tomando el límite de la rama más alta como la altura máxima.

Las especies fueron identificadas in situ. No obstante, los taxones desconocidos o de los que existieron dudas, se recolectaron muestras para su posterior identificación por medio de claves dicotómicas de floras del estado de Aguascalientes y Jalisco (González, 1986; De la Cerda, 1999; Siqueiros, 1999), en el herbario de la Universidad Autónoma de Aguascalientes (HUAA).

\section{Análisis de la información}

La riqueza encontrada y la eficiencia del muestreo se evaluaron mediante curvas de acumulación de especies. Esto se realizó a partir de los datos de la abundancia de las especies encontradas en los sitios muestreados y por medio de los estimadores no paramétricos ACE, Chao-1, Jackknife y Bootstrap. Dichos índices, basados en la abundancia de las especies por sitio, cuantifican la cantidad de especies de baja densidad que pudieron haber sido omitidas por el muestreo (Colwell et al., 2012). Estos estimadores fueron generados con el programa EstimateS v. 9.1 (Colwell, 2016).

\section{Diversidad}

Con los datos de abundancia recabados sobre las especies presentes en cada sitio, se realizó un agrupamiento para definir las asociaciones vegetales en la zona de estudio. Lo anterior se realizó por medio de un análisis cluster. Este análisis fue realizado mediante el programa PC ORD v. 6.0 (McCune y Mefford, 2011) y se consideró como medida de distancia el índice de Sørensen (Bray-Curtis) y el promedio grupal como método de ligamiento. La distancia de corte se definió conforme a lo observado en campo (composición y fisonomía), para ajustar el agrupamiento a la realidad actual observada en Monte Grande.

La diversidad alfa de cada sitio y la asociación se estimaron mediante el número efectivo de especies (Jost, 2006). Esta forma de expresar la diversidad permite comparar la magnitud en la diferencia de diversidad de dos o más comunidades (Moreno et al., 2011). Se empleó la diversidad verdadera de orden cero $\left({ }^{\circ} D\right)$ que equivale a la riqueza de especies $\left({ }^{\circ} D=S\right)$, ya que es insensible a la abundancia de los individuos. Al igual que se utilizó la diversidad verda- dera de orden $1\left({ }^{1} D\right)$ y orden $2\left({ }^{2} D\right)$, donde se considera la abundancia proporcional de cada especie. La diversidad de orden 1 se puede calcular directamente a partir de la expresión del índice de Shannon, ${ }^{1} D=\exp \left(H^{\prime}\right)$; mientras que la de orden 2 se obtiene a partir del índice de Simpson, ${ }^{2} D=1 / S^{\prime}$ (Jost, 2006; Moreno et al., 2011).

\section{Estructura}

Las características estructurales de la vegetación se determinaron calculando la densidad de las especies (ind/ha), definida como el número de individuos en el área de muestreo; dominancia a partir del área basal ( $\mathrm{m}^{2} / \mathrm{ha}$ ) y frecuencia (número de veces que cada especie aparece en los sitios). Se obtuvieron datos de altura promedio de todos los individuos por sitio (0.03 ha) y la altura máxima $(\mathrm{m})$. Esta última se estimó tomando al individuo más alto de cada muestreo (Zacarías-Eslava et al., 2011). El área basal se determinó de la siguiente manera (Mueller-Dombois y Ellenberg, 1974):

$$
A B=\pi(d / 2) 2
$$

donde $d$ representa el diámetro y $\pi$ la constante 3.1416. Para conocer la importancia ecológica de las especies registradas en cada asociación del sitio de estudio, se calculó el valor de importancia relativa (Mueller-Dombois y Ellenberg, 1974; Zacarías-Eslava et al., 2011):

$$
V I R=(F R+D R+A B R) / 3
$$

donde FR representa la frecuencia relativa; $D R$, la densidad relativa y $A B R$, el área basal relativa.

\section{Análisis estadístico}

Se evaluó si existieron diferencias entre las asociaciones muestreadas en cuanto a variables de diversidad de especies, así como variables estructurales (densidad, área basal y altura). Para lo anterior se realizaron pruebas de ShapiroWilk para comprobar el supuesto estadístico de normalidad. Se realizó una prueba de análisis de varianza (ANOVA) de una vía $(\mathrm{P} \leq 0.05)$ cuando los datos presentaron una distribución normal, en caso contrario (datos sin una distribución normal) se procedió a realizar una prueba no paramétrica de Kruskal-Wallis ( $\mathrm{P} \leq 0.05)$. Al presentar diferencias significativas las pruebas anteriores se hicieron comparaciones múltiples mediante la prueba Tukey HSD ( $\mathrm{P} \leq 0.05)$, para variables que cumplieron el supuesto de normalidad, y una comparación por pares mediante de una prueba no 
paramétrica de Kruskal-Wallis $(\mathrm{P} \leq 0.05)$, para variables que no cumplieron el supuesto. Esto para conocer cuáles atributos evaluados difieren o no entre cada una de las asociaciones identificadas. Lo anterior se realizó en el programa InfoStat v. 2016 (Di-Rienzo et al., 2016).

\section{Resultados}

\section{Composición y diversidad}

Se registraron 26 especies en nueve géneros y ocho familias. Las familias mejor representadas en cuanto al número de especies fueron Fagaceae (nueve especies), Pinaceae (seis) y Ericaceae (cuatro). Los géneros mejor representados fueron
Quercus (nueve especies), Pinus L. (seis), Arbutus L. (tres) y Juniperus L. (tres), los demás incluyen una especie (Cuadro 2). El número total de especies registradas en las unidades de muestreo fue menor al predicho por la curva de acumulación de especies construida con estimadores no paramétricos. Los estimadores indicaron que el muestreo de especies representó entre $87 \%$ (Jackknife) y $95 \%$ (ACE) del número total potencialmente presente en Monte Grande (Fig. 2).

El análisis de agrupamiento muestra una vegetación heterogénea. Se obtuvieron cinco asociaciones (denominadas por los elementos dominantes). La primera separación se presenta con el grupo de mayor presencia de especies

Cuadro 2: Listado de especies registradas en Monte Grande, dentro del Área Natural Protegida Sierra Fría, Aguascalientes, México.

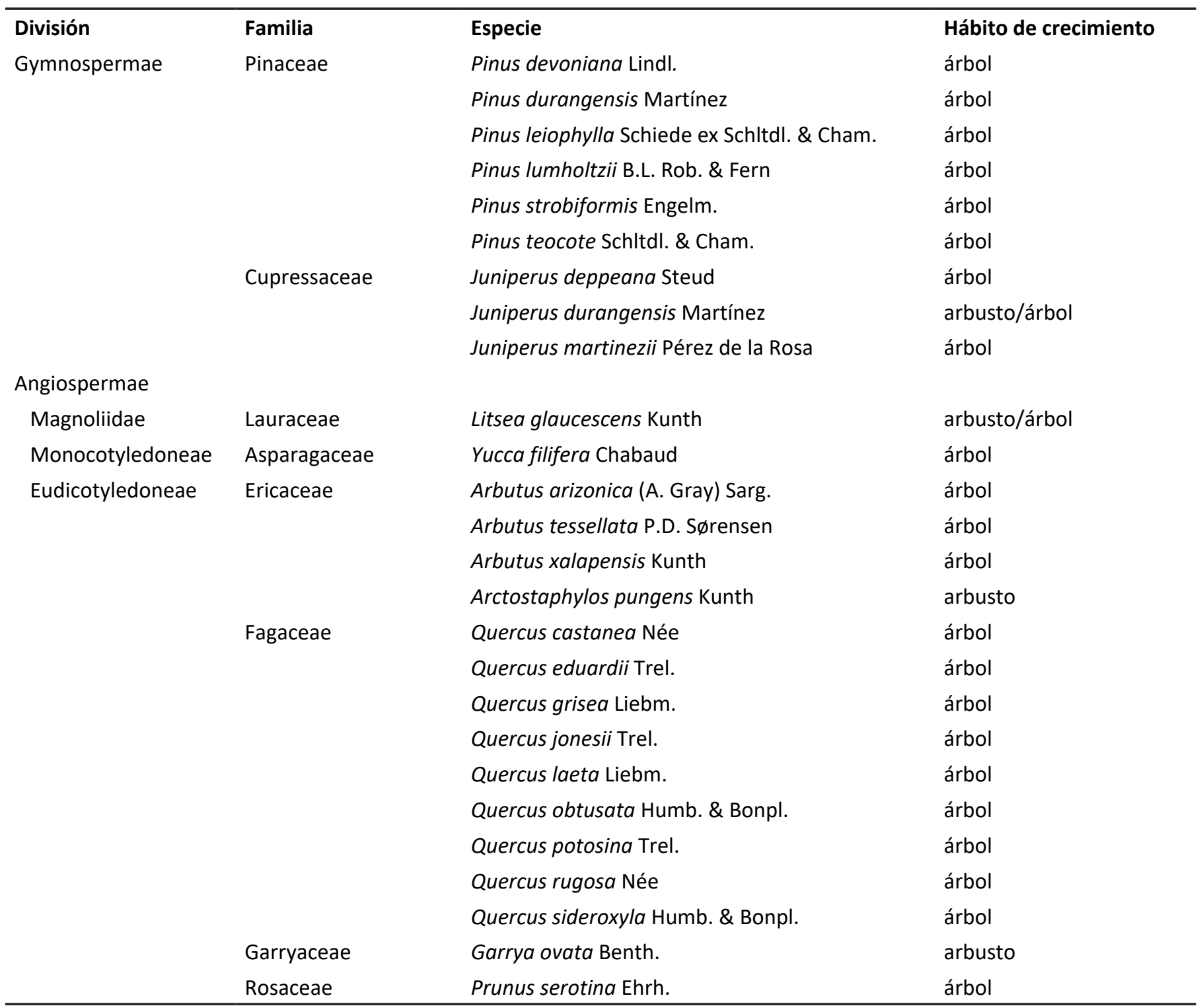




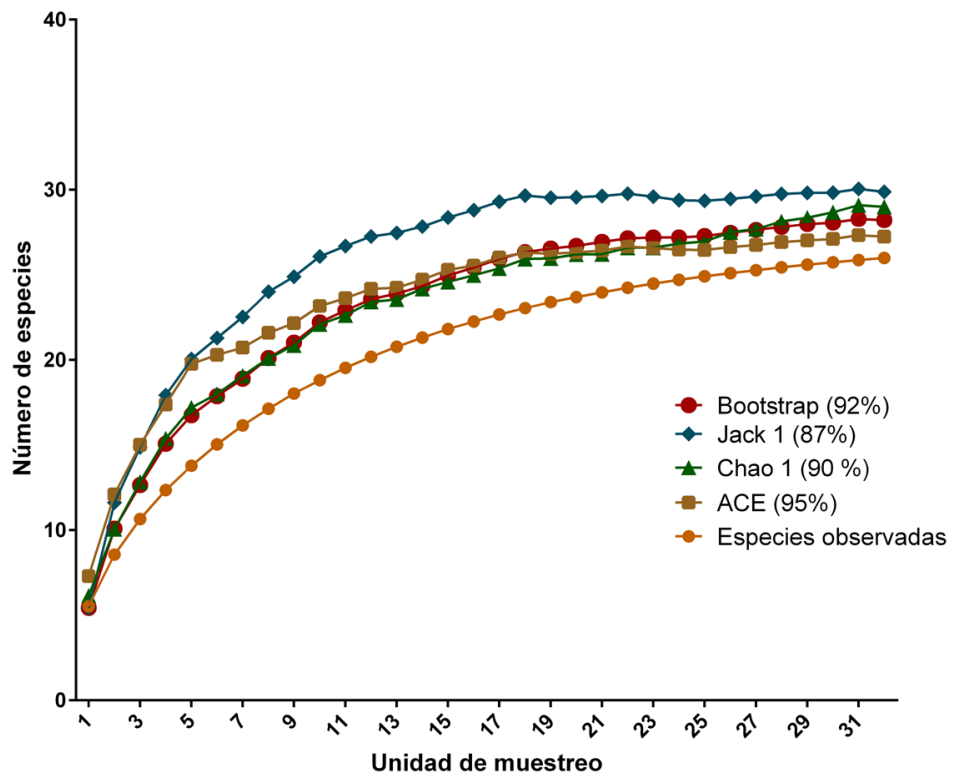

Figura 2: Curvas de acumulación de especies generadas con 32 sitios muestreados en Monte Grande, dentro del Área Natural Protegida Sierra Fría, Aguascalientes, México. Las curvas fueron realizadas según las especies observadas, su abundancia y estimadores no paramétricos basados en la abundancia. Se presenta el valor de complementariedad (porcentaje del número total de especies registrado en los 32 sitios de muestreo, respecto de aquel esperado por los estimadores no-paramétricos).

de Pinus (5), la segunda separación entre los sitios con mayor dominancia de Juniperus ( 3 y 4 ) y Quercus ( 1 y 2 ), con una similitud de $23.7 \%$. En el grupo de los Quercus se separan a $35.9 \%$ de similitud por dominancia de $Q$. sideroxyla y $Q$. potosina. Las cinco asociaciones observadas fueron: (1) bosque de encino Quercus sideroxyla-Quercus rugosa, (2) bosque de encino Quercus potosina-A. pungens, (3) bosque mixto de Juniperus-Quercus, (4) bosque de Juniperus, (5) bosque mixto de Quercus-Pinus (Fig. 3). Los bosques de encino $Q$. sideroxyla- $Q$. rugosa y de encino $Q$. potosina- $A$.

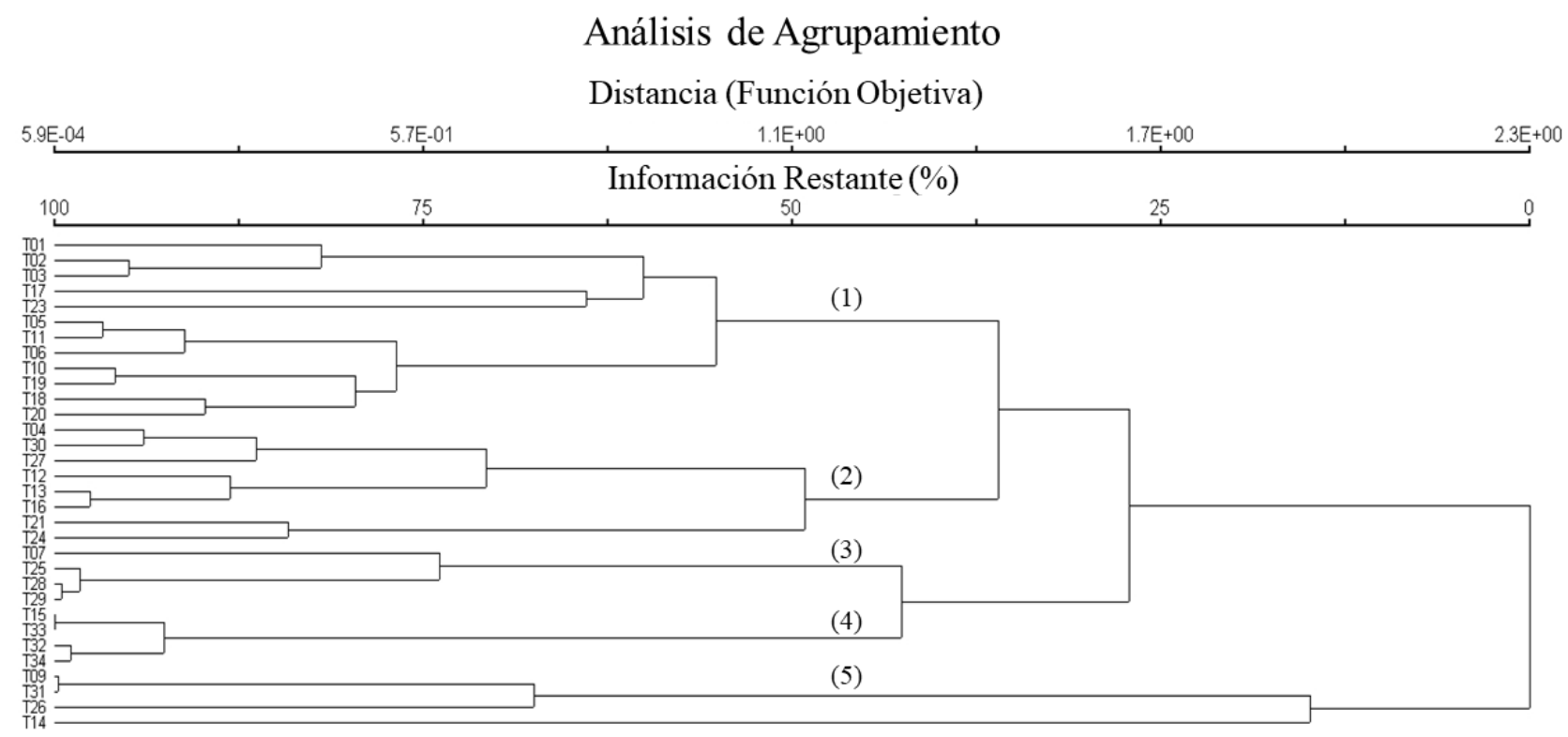

Figura 3: Dendrograma que muestra el agrupamiento de cinco asociaciones vegetales identificadas en Monte Grande, dentro del Área Natural Protegida Sierra Fría, Aguascalientes, México: 1. Bosque de encino Quercus sideroxyla-Q. rugosa; 2. Bosque de encino Q. potosina-Arctostaphylos pungens; 3. Bosque mixto de Juniperus-Quercus; 4. Bosque de Juniperus; 5. Bosque mixto de Quercus-Pinus. Usando como medida de similitud el índice de Sørensen (Bray-Curtis). 
pungens tuvieron la mayor distribución en la zona de estudio. El bosque de $Q$. sideroxyla- $Q$. rugosa se encontró principalmente a altitudes mayores a $2800 \mathrm{~m}$ y a menor altitud en fondo de cañadas. El bosque de $Q$. potosina-A. pungens figura a 2600-3000 m, en sitios con suelos someros, alta pendiente y exposiciones sur. Las tres asociaciones restantes presentaron distribución restringida. El bosque de Juniperus se presentó a altitudes mayores a $2800 \mathrm{~m}$ en sitios de poca pendiente y suelos profundos; mientras que el bosque de Juniperus-Quercus en altitudes menores a 2600 $\mathrm{m}$, de la misma forma en sitios de poca pendiente y suelos profundos. El bosque mixto de Quercus-Pinus mayormente se observó entre $2600-2800$ m s.n.m. en suelos someros y calcáreos.

La diversidad de especies fue significativamente diferente entre asociaciones, tanto en riqueza $(F=4.83$, g.l. $=4$, $\mathrm{P}=0.0047)$, diversidad de orden $1(\mathrm{~F}=4.34$, g. $\mathrm{I}=4, \mathrm{P}=0.0080)$ y orden 2 ( $F=3.26$, g.I. $=4, P=0.0272)$. El bosque mixto de Quercus-Pinus, seguido del bosque de $Q$. sideroxyla- $Q$. rugosa y bosque de $Q$. potosina- $A$. pungens, presentó la mayor diversidad. En el bosque de Juniperus-Quercus y el bosque de Juniperus se encontró el menor valor de diversidad (Cuadro 3). Esto representa, en número de especies efectivas que el bosque de Juniperus es 2.68 veces menos diverso que el bosque mixto de Quercus-Pinus y 2.42 veces menos diverso que los bosques de Quercus.

\section{Estructura}

La densidad ( $\mathrm{H}=12.81$, g.I. $=4, \mathrm{P}=0.0121)$, el área basal $(H=9.54, g . I .=4, P=0.048)$, y la altura promedio $(H=22.50$, g.I. $=4, P=0.0002$ ) fueron significativamente diferentes entre las asociaciones identificadas (Cuadro 3 ). El bosque de Quercus-Pinus, seguido del bosque de $Q$. potosina- $A$. pungens y $Q$. sideroxyla- $Q$. rugosa, presentaron la mayor densidad. El bosque de Juniperus y Juniperus-Quercus presentaron los valores más bajos en esta variable.

En el área basal solo se presentaron diferencias significativas entre el bosque de $Q$. sideroxyla- $Q$. rugosa y el bosque de $Q$. potosina-A. pungens, teniendo el valor mayor y menor respectivamente. Las asociaciones presentaron una distribución diamétrica principalmente en las primeras clases diamétricas, de 5 a $25 \mathrm{~cm}$ (Fig. 4). Las especies con mayor diámetro promedio pertenecen a los géneros Arbutus y Pinus, y las de menor diámetro medio son arbustos como Arctostaphylos pungens y Garrya ovata Benth.

Cuatro de las asociaciones encontradas no difieren estadísticamente en su altura; únicamente el bosque de encino $Q$. sideroxyla- $Q$. rugosa presenta diferencias significativas, con valores de altura promedio mayores. Las especies que alcanzaron mayor altura en individuos viejos son Q. sideroxyla (18 m), Q. rugosa (15 m), Arbutus xalapensis Kunth (14 m) y Pinus durangensis Martínez (14 m), dentro de las asociaciones donde se llegan a observar. En las cin-

Cuadro 3: Valores promedio ( \pm desviación estándar) de diversidad y estructura para las asociaciones identificadas a partir del análisis de agrupamiento en Monte Grande, dentro del Área Natural Protegida Sierra Fría, Aguascalientes, México. Los valores con una letra común no son significativamente diferentes $(P>0.05)$, de acuerdo con la prueba de Tukey para variables de diversidad y Kruskal-Wallis para variables estructurales.

\begin{tabular}{|c|c|c|c|c|c|}
\hline Asociación vegetal & $\begin{array}{l}\text { Bosque de Juniperus } \\
\text { (BJ) }\end{array}$ & $\begin{array}{l}\text { Bosque mixto de } \\
\text { Juniperus-Quercus } \\
\text { (BJQ) }\end{array}$ & $\begin{array}{l}\text { Bosque de encino } \\
\text { Quercus potosina- } \\
\text { Arctostaphylos } \\
\text { pungens (BQC) }\end{array}$ & $\begin{array}{l}\text { Bosque mixto de } \\
\text { Quercus-Pinus } \\
\text { (BQP) }\end{array}$ & $\begin{array}{l}\text { Bosque de encino } \\
\text { Quercus sideroxyla- } \\
\text { Quercus rugosa } \\
\text { (BQS) }\end{array}$ \\
\hline Número de sitios & 4 & 4 & 8 & 3 & 12 \\
\hline \multicolumn{6}{|l|}{ Diversidad } \\
\hline Riqueza promedio $(S)$ & $2.75 \pm 0.96 \mathrm{a}$ & $4.25 \pm 0.50 \mathrm{ab}$ & $6.13 \pm 2.95 b$ & $7.00 \pm 2.00 \mathrm{~b}$ & $6.42 \pm 1.44 b$ \\
\hline Especies efectivas $\left({ }^{1} D\right)$ & $1.50 \pm 0.26 \mathrm{a}$ & $2.52 \pm 0.59 a b$ & $3.64 \pm 1.82 \mathrm{~b}$ & $4.03 \pm 1.11 b$ & $3.86 \pm 1.17 b$ \\
\hline Especies efectivas $\left({ }^{2} D\right)$ & $1.27 \pm 0.13 \mathrm{a}$ & $2.14 \pm 0.71 a b$ & $3.08 \pm 1.57 \mathrm{ab}$ & $3.24 \pm 0.88 b$ & $3.12 \pm 1.18 a b$ \\
\hline \multicolumn{6}{|l|}{ Datos estructurales } \\
\hline Densidad (ind/ha) & $867 \pm 221 a$ & $992 \pm 307 a$ & $1622 \pm 498 b$ & $2200 \pm 600 \mathrm{~b}$ & $1278 \pm 649 a b$ \\
\hline Área basal (m²/ha) & $37.58 \pm 14.6 \mathrm{ab}$ & $36.08 \pm 18.3 \mathrm{ab}$ & $36.41 \pm 8 a$ & $37.11 \pm 9.3 \mathrm{ab}$ & $53.56 \pm 29.3 b$ \\
\hline Altura promedio (m) & $3.65 \pm 0.44$ a & $4.05 \pm 0.45 \mathrm{a}$ & $4.62 \pm 1.29 \mathrm{a}$ & $4.00 \pm 0.23 \mathrm{a}$ & $8.57 \pm 1.61 b$ \\
\hline Altura máxima (m) & 9 & 14 & 13 & 14 & 18 \\
\hline
\end{tabular}




\section{Bosque de Juniperus}

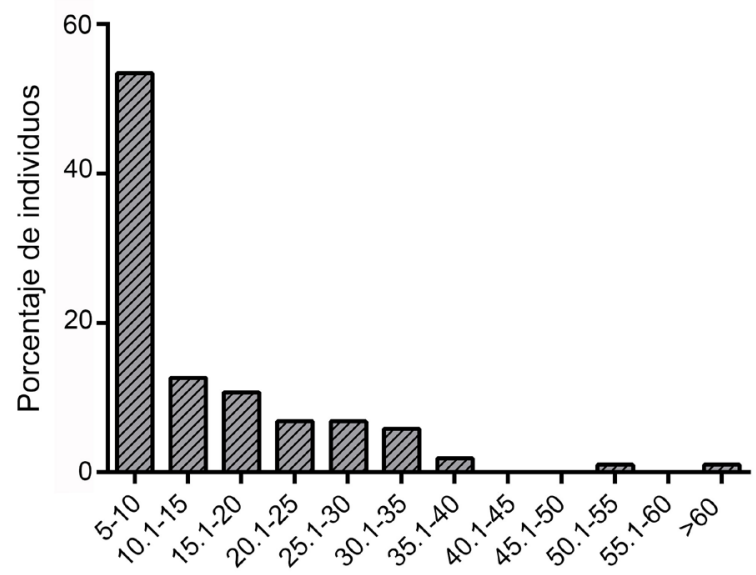

Clase diamétrica $(\mathrm{cm})$

Bosque de encino Quercus sideroxyla-Quercus rugosa

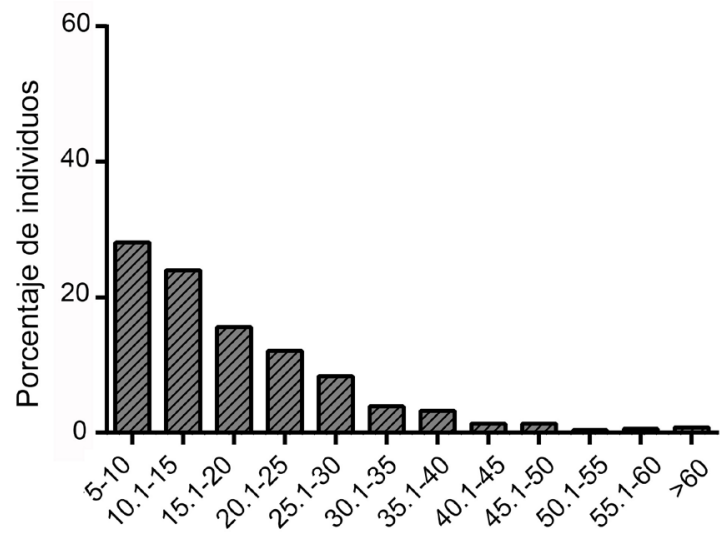

Clase diamétrica $(\mathrm{cm})$

\section{Bosque mixto de Quercus-Pinus}

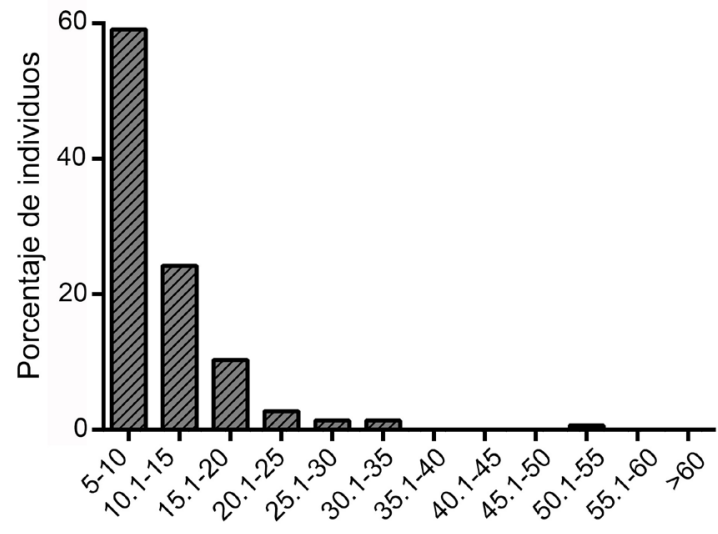

Clase diamétrica $(\mathrm{cm})$

\section{Bosque de encino Quercus potosina-Arctostaphylos pungens}

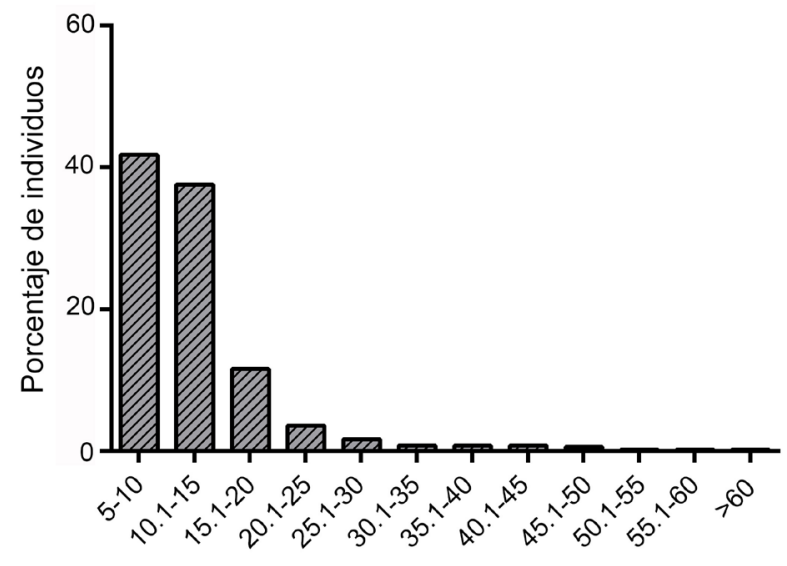

Clase diamétrica $(\mathrm{cm})$

\section{Bosque mixto de Juniperus-Quercus}

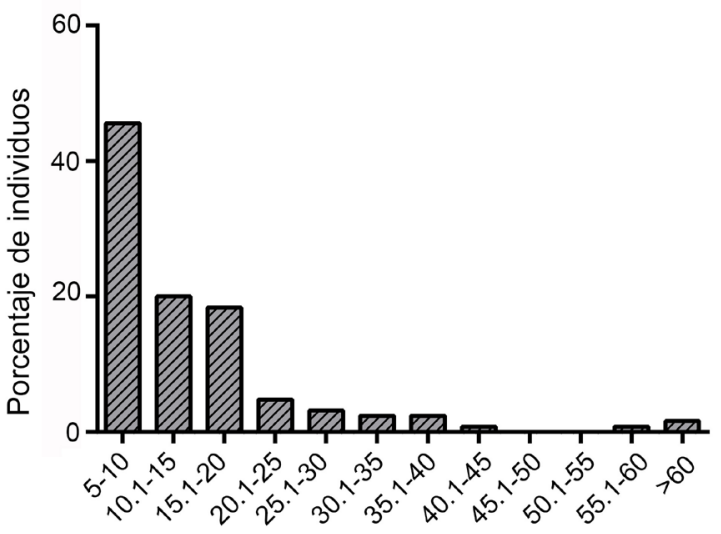

Clase diamétrica $(\mathrm{cm})$

Figura 4: Distribución de la estructura horizontal por tipo de asociación vegetal de Monte Grande, dentro del Área Natural Protegida Sierra Fría, Aguascalientes, México. 
co asociaciones la especie que presentó menor altura fue A. pungens (Cuadro 4). En el caso del bosque de encino $Q$. sideroxyla-Q. rugosa, el estrato arbustivo es más escaso, no obstante, en cañadas húmedas, las especies de menor altura fueron Prunus serotina Ehrh. y en ocasiones Litsea glaucescens Kunth.
Las especies con mayor valor de importancia relativa (VIR) son similares entre las asociaciones vegetales, como es el caso de A. pungens, J. deppeana, $Q$. sideroxyla y $Q$. potosina, que son especies de amplia distribución en la zona de estudio (Cuadro 4). Otras especies que llegan a tener un VIR alto son $Q$. jonesii, $P$. lumholtzii y $P$. durangensis

Cuadro 4: Valores estructurales y de Importancia Relativa en bosques templados de Monte Grande, dentro del Área Natural Protegida Sierra Fría, Aguascalientes, México. Alt (altura \pm desviación estándar), D/ha (densidad ind/ha), Dr (densidad relativa), AB/ha (área basal $\mathrm{m}^{2} / \mathrm{ha}$ ), Abr (área basal relativa), $\mathrm{F}$ (frecuencia), Fr (frecuencia relativa), VIR (Valor de importancia relativa). Los tres valores más altos de VIR se muestran en negritas.

\begin{tabular}{|c|c|c|c|c|c|c|c|c|}
\hline Asociación/Especie & Alt & D/ha & $\mathrm{Dr}$ & $A B / h a$ & Abr & $\mathbf{F}$ & $\mathrm{Fr}$ & VIR \\
\hline \multicolumn{9}{|l|}{ Bosque de encinos $Q$. potosina- $A$. pungens } \\
\hline Quercus potosina Trel. & $4.05 \pm 1.60$ & 871 & 52.25 & 13.25 & 40.50 & 8 & 16.67 & 36.47 \\
\hline Arctostaphylos pungens Kunth & $2.79 \pm 1.03$ & 446 & 26.75 & 5.25 & 16.05 & 8 & 16.67 & 19.82 \\
\hline Juniperus deppeana Steud & $5.40 \pm 1.41$ & 83 & 5.00 & 3.46 & 10.57 & 6 & 12.50 & 9.36 \\
\hline Quercus sideroxyla Humb. \& Bonpl. & $6.76 \pm 3.24$ & 100 & 6.00 & 3.50 & 10.70 & 5 & 10.42 & 9.04 \\
\hline Quercus rugosa Née & $7.06 \pm 3.20$ & 75 & 4.50 & 4.29 & 13.12 & 3 & 6.25 & 7.96 \\
\hline Arbutus xalapensis Kunth & $5.50 \pm 2.65$ & 21 & 1.25 & 1.29 & 3.95 & 3 & 6.25 & 3.82 \\
\hline Arbutus arizonica (A. Gray) Sarg. & $6.88 \pm 2.53$ & 13 & 0.75 & 0.83 & 2.55 & 3 & 6.25 & 3.18 \\
\hline Prunus serotina Ehrh. & $4.23 \pm 1.54$ & 13 & 0.75 & 0.13 & 0.38 & 2 & 4.17 & 1.77 \\
\hline Otros (8) & & 46 & 2.75 & 0.72 & 2.19 & 10 & 20.83 & 8.59 \\
\hline Total & & 1622 & & 36.41 & & 55 & & 100 \\
\hline \multicolumn{9}{|l|}{ Bosque de encino Q. sideroxyla-Q. rugosa } \\
\hline Quercus sideroxyla Humb. \& Bonpl. & $10.7 \pm 3.6$ & 633 & 49.57 & 34.39 & 64.20 & 12 & 14.86 & 43.12 \\
\hline Quercus rugosa Née & $7.49 \pm 3.71$ & 211 & 16.52 & 7.75 & 14.47 & 9 & 9.46 & 14.23 \\
\hline Arbutus xalapensis Kunth & $9.79 \pm 2.10$ & 89 & 6.96 & 4.44 & 8.30 & 8 & 10.81 & 8.55 \\
\hline Juniperus deppeana Steud & $6.29 \pm 1.61$ & 75 & 5.87 & 1.83 & 3.42 & 9 & 12.16 & 6.99 \\
\hline Arctostaphylos pungens Kunth & $2.57 \pm 1.03$ & 78 & 6.09 & 0.42 & 0.78 & 8 & 10.81 & 5.75 \\
\hline Quercus obtusata Humb. \& Bonpl. & $6.02 \pm 2.58$ & 69 & 5.43 & 1.94 & 3.63 & 5 & 6.76 & 5.19 \\
\hline Quercus potosina Trel. & $4.72 \pm 1.69$ & 56 & 4.35 & 0.67 & 1.24 & 6 & 8.11 & 4.46 \\
\hline Arbutus tessellata P.D. Sørensen & $6.96 \pm 3.72$ & 22 & 1.74 & 0.89 & 1.66 & 5 & 6.76 & 3.30 \\
\hline Otros (10) & & 44 & 3.48 & 1.23 & 2.29 & 15 & 20.27 & 8.42 \\
\hline Total & & 1278 & & 53.56 & & 74 & & 100 \\
\hline \multicolumn{9}{|l|}{ Bosque mixto de Quercus-Pinus } \\
\hline Quercus jonesii Trel. & $3.44 \pm 1.49$ & 967 & 43.94 & 14.89 & 40.12 & 3 & 13.64 & 32.57 \\
\hline Arctostaphylos pungens Kunth & $1.77 \pm 0.53$ & 522 & 23.74 & 6.56 & 17.66 & 3 & 13.64 & 18.35 \\
\hline Pinus lumholtzii B.L. Rob. \& Fern & $5.19 \pm 1.85$ & 189 & 8.59 & 3.89 & 10.48 & 3 & 13.64 & 10.90 \\
\hline Pinus teocote Schltdl. \& Cham. & $4.43 \pm 1.48$ & 178 & 8.08 & 2.22 & 5.99 & 3 & 13.64 & 9.24 \\
\hline Pinus durangensis Martínez & $3.93 \pm 2.61$ & 111 & 5.05 & 3.89 & 10.48 & 2 & 9.09 & 8.21 \\
\hline Pinus leiophylla Schiede ex Schltdl. \& Cham. & $4.13 \pm 2.72$ & 89 & 4.04 & 2.78 & 7.49 & 2 & 9.09 & 6.87 \\
\hline Pinus strobiformis Engelm. & $5.43 \pm 1.99$ & 78 & 3.54 & 1.44 & 3.89 & 1 & 4.55 & 3.99 \\
\hline Juniperus durangensis Martínez & $2.33 \pm 0.58$ & 33 & 1.52 & 0.22 & 0.60 & 2 & 9.09 & 3.73 \\
\hline Otros (2) & & 33 & 1.52 & 1.22 & 3.29 & 3 & 13.64 & 6.15 \\
\hline Total & & 2200 & & 37.11 & & 22 & & 100 \\
\hline
\end{tabular}


Cuadro 4. Continuación.

\begin{tabular}{|c|c|c|c|c|c|c|c|c|}
\hline Asociación/Especie & Alt & D/ha & $\mathrm{Dr}$ & $A B / h a$ & Abr & $\mathbf{F}$ & $\mathrm{Fr}$ & VIR \\
\hline \multicolumn{9}{|l|}{ Bosque mixto de Juniperus-Quercus } \\
\hline Juniperus deppeana Steud & $3.88 \pm 2.30$ & 592 & 59.66 & 22.58 & 62.59 & 4 & 23.53 & 48.59 \\
\hline Arctostaphylos pungens Kunth & $2.65 \pm 0.72$ & 192 & 19.33 & 2.58 & 7.16 & 3 & 17.65 & 14.71 \\
\hline Quercus potosina Trel. & $5.19 \pm 0.99$ & 83 & 8.40 & 4.17 & 11.55 & 4 & 23.53 & 14.49 \\
\hline Quercus grisea Liebm. & $4.36 \pm 1.03$ & 75 & 7.56 & 2.50 & 6.93 & 3 & 17.65 & 10.71 \\
\hline Yucca filifera Chabaud & $6.17 \pm 0.29$ & 25 & 2.52 & 3.67 & 10.16 & 1 & 5.88 & 6.19 \\
\hline Quercus eduardii Trel. & $7.0 \pm 0.2$ & 17 & 1.68 & 0.25 & 0.69 & 1 & 5.88 & 2.75 \\
\hline Quercus laeta Liebm. & $8.0 \pm 0.5$ & 8 & 0.84 & 0.33 & 0.92 & 1 & 5.88 & 2.55 \\
\hline Total & & 992 & & 36.08 & & 17 & & 100 \\
\hline \multicolumn{9}{|l|}{ Bosque de Juniperus } \\
\hline Juniperus deppeana Steud & $3.40 \pm 1.41$ & 775 & 89.42 & 28.00 & 74.50 & 4 & 33.33 & 65.75 \\
\hline Quercus sideroxyla Humb. \& Bonpl. & $6.13 \pm 0.85$ & 50 & 5.77 & 7.67 & 20.40 & 4 & 33.33 & 19.83 \\
\hline Arctostaphylos pungens Kunth & $2.60 \pm 0.46$ & 25 & 2.88 & 0.25 & 0.67 & 3 & 25 & 9.52 \\
\hline Pinus teocote Schltdl. \& Cham. & $5.6 \pm 3.66$ & 17 & 1.92 & 1.67 & 4.43 & 1 & 8.33 & 4.90 \\
\hline Total & & 867 & & 37.58 & & 12 & & 100 \\
\hline
\end{tabular}

en los bosques de Quercus-Pinus. En el bosque de encino $Q$. sideroxyla-Q. rugosa, además de $Q$. sideroxyla como la especie con mayor VIR, se encuentra $Q$. rugosa en la segunda posición y $A$. xalapensis en tercer lugar. Estas especies tienen una distribución diamétrica en las primeras clases $(<15 \mathrm{~cm})$, con poblaciones en regeneración como es el caso de $Q$. jonesii Trel. y $P$. durangensis (Fig. 5), aunque se llegan a observar individuos con diámetros mayores.

\section{Discusión}

\section{Composición y diversidad}

La composición de plantas leñosas registradas para el área estudiada es representativa de un bosque templado, en cuanto a las familias mejor representadas (Fagaceae, Ericaceae, Pinaceae y Cupressaceae) (Rzedowski, 2006). Los bosques estudiados presentan una composición arbórea y arbustiva, que por su ubicación en la zona sur de la Sierra Madre Occidental y cercanía a otras provincias fisiográficas como son el Altiplano sur y el Eje Neovolcánico, integra taxones que se han reportado en distintas provincias fisiográficas. Monte Grande comparte 10 especies arbóreas con los bosques de Quercus de la Sierra de Santa Rosa, Guanajuato (Altiplano sur) (Martínez-Cruz et al., 2009) y comparte 15 con bosques templados de Pinus-Quercus en Durango (Sierra Madre Occidental) (Márquez-Linares et al.,
1999; Návar-Cháidez y González-Elizondo, 2009). Los bosques dominados por $Q$. sideroxyla y los de Quercus-Pinus son más semejantes en composición florística a los de Durango, mientras que los bosques de $Q$. potosina lo son más a los de Guanajuato, posiblemente debido a que comparte similitudes ambientales entre los sitios, como precipitación $(650-800 \mathrm{~mm})$ y temperatura promedio $\left(12-18^{\circ} \mathrm{C}\right)$.

Por otra parte, con 26 especies registradas, Monte Grande presenta una mayor riqueza que la reportada para bosques templados de "El Salto", Durango (18 especies), donde se midieron todas las especies en parcelas de 100 $\mathrm{m}^{2}$ (Domínguez-Gómez et al., 2018). Fue similar al reportado por Márquez-Linares et al. (1999) en bosques de tres localidades de San Dimas, Durango (27 especies), donde se midieron, igual que en este estudio, individuos con diámetro de $5 \mathrm{~cm}$ en parcelas de $500 \mathrm{~m}^{2}$. Estos sitios presentan similitud climática (templado subhúmedo) y pertenecen a la misma provincia fisiográfica (Sierra Madre Occidental), solo siendo diferente el manejo que llega a tener cada bosque. En el caso de "El Salto", Durango, puede que esté influenciado por el tratamiento silvícola que tiene, posiblemente presentado una poda selectiva que favorece la presencia de pocas especies (González-Espinosa et al., 1991; Domínguez-Gómez et al., 2018). Por otra parte, la riqueza es menor a la encontrada en bosques de Quercus en la Sierra de 


\section{Quercus sideroxyla}

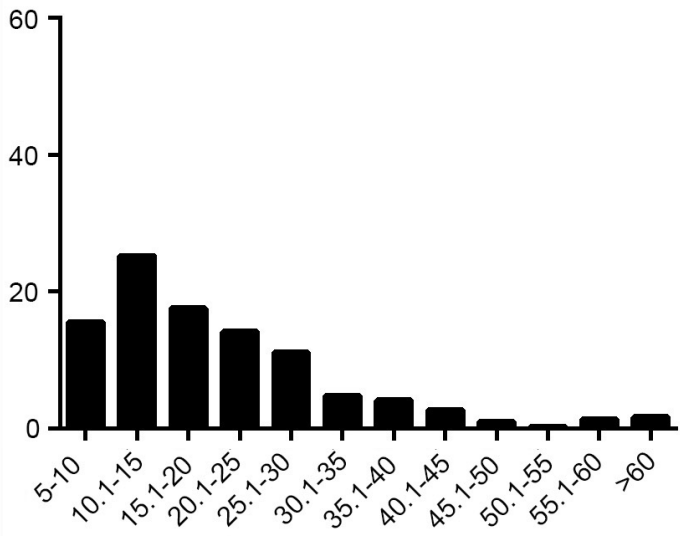

Clase diamétrica $(\mathrm{cm})$

Quercus jonesii

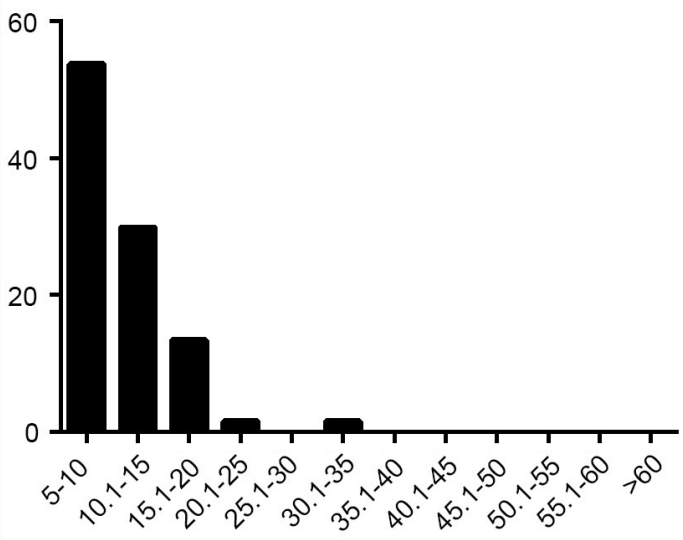

Clase diamétrica $(\mathrm{cm})$

Juniperus deppeana

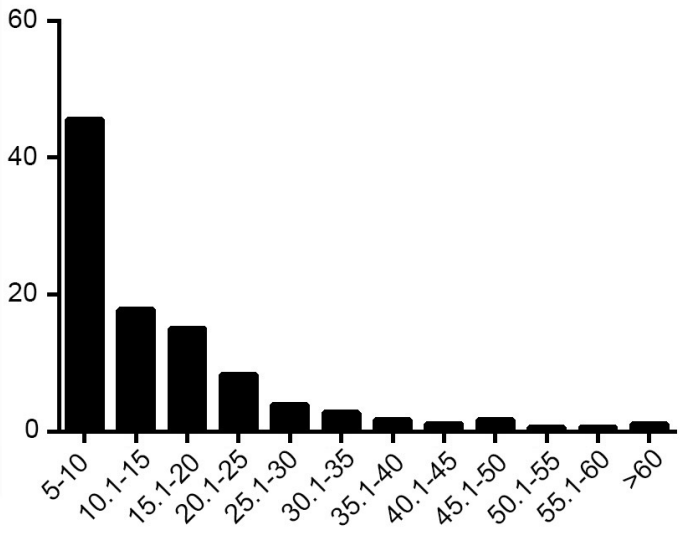

Clase diamétrica $(\mathrm{cm})$

\section{Quercus potosina}

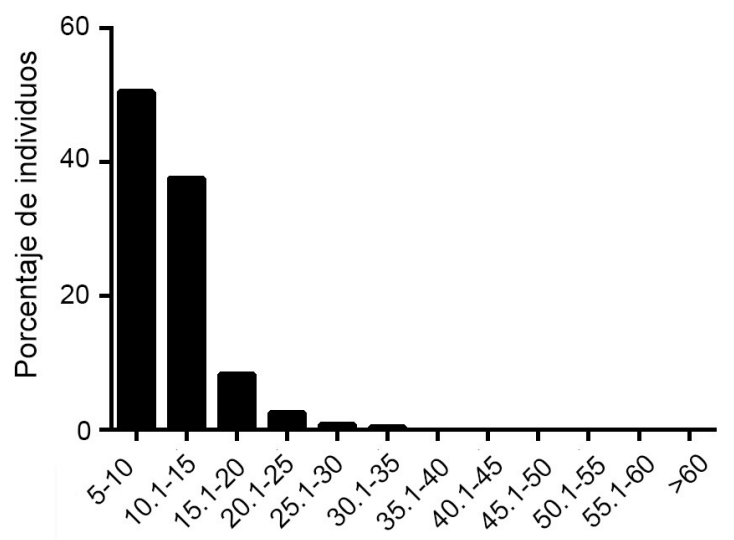

Clase diamétrica $(\mathrm{cm})$

\section{Quercus rugosa}

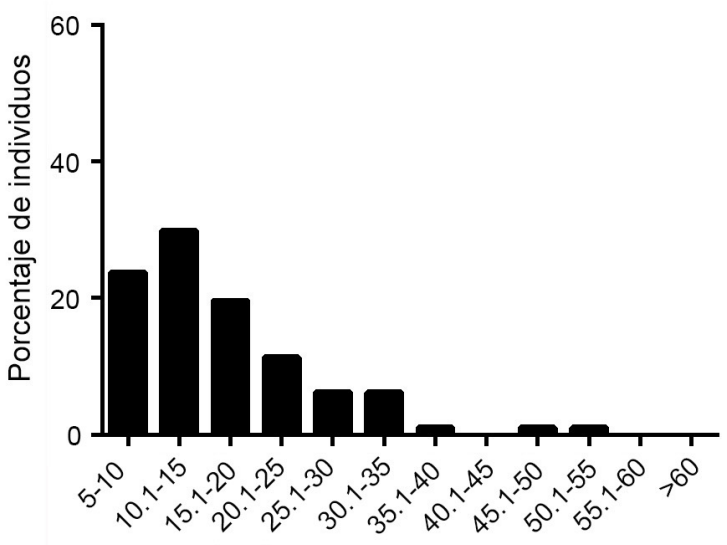

Clase diamétrica $(\mathrm{cm})$

\section{Pinus durangensis}

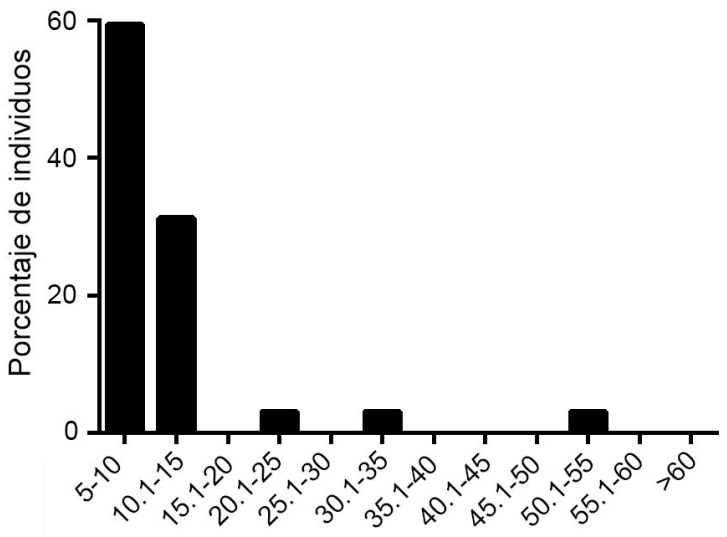

Clase diamétrica $(\mathrm{cm})$

Figura 5: Distribución diamétrica de algunas de las especies arbóreas más importantes en Monte Grande, dentro del Área Natural Protegida Sierra Fría, Aguascalientes, México. Las especies fueron elegidas por presentar valores altos de valor de importancia relativa (VIR). 
Manantlán (34 especies) (Olvera-Vargas y Figueroa-Rangel, 2012). En este caso se usó la misma categoría diamétrica (5 $\mathrm{cm}$ ) y parcelas de $500 \mathrm{~m}^{2}$, la variación en riqueza se puede deber a que el sitio se ve más favorecido con mayor lluvia (1200-1300 mm) y una menor altitud (2000-2450 m).

En los bosques estudiados, la diversidad encontrada sugiere que son bosques poco diversos, aunque esto es común en bosques templados de México. Los valores aquí encontrados (1.50-3.86 $\left.{ }^{1} D\right)$ son comparables a otros bosques templados del país, transformando la diversidad encontrada en otros estudios de Shannon-Wiener a especies efectivas $\left({ }^{1} D=\exp \left(H^{\prime}\right)\right)$. Siendo similares a los bosques templados de Durango reportados por Návar-Cháidez y González-Elizondo (2009) donde se mencionan valores de 0.531.33 $H^{\prime}\left(1.69-3.78{ }^{1} D\right)$, y a los reportados por Martínez-Cruz et al. (2009) donde reportan valores de $1.034 \mathrm{H}^{\prime}\left(2.81^{1} \mathrm{D}\right)$ para bosques de Quercus en Santa Rosa, Guanajuato. En comparación con la diversidad de las zonas más bajas del ANPSF resulta ser menor en algunos casos, reportando diversidades que oscilan entre 0.81-1.63 $H^{\prime}\left(2.24-5.10{ }^{1} D\right)$ (Díaz-Núñez et al., 2012).

Los bosques templados son menos diversos en comparación con los bosques tropicales $\left(3.35 \mathrm{H}^{\prime}, 28.50{ }^{1} \mathrm{D}\right)$ (Chazdon, 2003; Maldonado-Sánchez y Maldonado-Mares, 2010). En los bosques templados la altitud es un factor que influye en la riqueza y diversidad de especies, conforme la altitud es mayor y las condiciones climáticas son menos favorables, la diversidad de especies es menor (Kappelle y Van Uffelen, 2006). Monte Grande presenta altitudes de 2480$3030 \mathrm{~m}$, esto puede tener influencia en la riqueza que se presentó en el sitio de estudio en comparación con otros sitios mencionados anteriormente, como es el caso de la Sierra de Manantlán con altitudes de 2000-2450 m (Olvera-Vargas y Figueroa-Rangel, 2012) y algunas de las zonas de menor altitud dentro del ANPSF (Díaz-Núñez et al., 2012; 2016).

\section{Estructura}

La fisonomía de los bosques estudiados coincide con lo reportado por Rzedowski (2006), quien menciona a los bosques de Juniperus como comunidades abiertas y de porte bajo (5-8 m). Al igual que menciona que la mayor parte de los bosques de Quercus en México están compuestos por comunidades en su mayoría de porte bajo y con alta densidad. Las densidades entre las asociaciones de Monte Grande varían de 867 a 2200 ind/ha, estos valores son menores, en su mayoría, a los reportados por DomínguezGómez et al. (2018), para bosques templados en Durango (2060 ind/ha) y mayores a las registradas por Olvera-Vargas y Figueroa-Rangel (2012), en bosques de Quercus de Sierra de Manantlán, Jalisco (512-887 ind/ha).

Los valores registrados de área basal en la asociación de bosque mixto de Quercus-Pinus $\left(37.11 \mathrm{~m}^{2} / \mathrm{ha}\right.$ ) presentan diferencias poco mayores con lo reportado por Márquez-Linares et al. (1999), donde se mencionan 24.87 a $26.82 \mathrm{~m}^{2} /$ ha en bosques de Pinus-Quercus en Durango. El área basal del bosque de $Q$. sideroxyla- $Q$. rugosa es comparable a la encontrada en los bosques dominados por $Q$. sideroxyla de Durango con $46.38 \mathrm{~m}^{2} /$ ha (Domínguez-Gómez et al., 2018). Esto es debido a que la composición de especies es parecida entre Monte Grande y los bosques estudiados en Durango. Las anteriores comparaciones muestran la alta variación en los bosques templados de México, posiblemente debido a las fluctuaciones climáticas y edáficas en las que se desarrollan (Rzedowski, 2006). Otro factor que no se puede dejar de mencionar es el papel del aprovechamiento con fines de producción económica y manejo, el cual es distinto en cada caso de acuerdo con la fisonomía y actividades en la región del bosque estudiado (Rzedowski, 2006; MartínezCruz et al., 2009).

En comparación con otras zonas previamente estudiadas en el ANPSF (en su mayoría zonas de 2400-2600 m), la composición y abundancia llega a ser distinta con Monte Grande, en especial en bosques de Quercus-Pinus y en bosques de Quercus. En el primer caso por la presencia de $Q$. jonesii y P. lumholtzii, que son poco comunes en las zonas bajas (Díaz-Núñez et al., 2012). En bosques de Quercus por la dominancia y amplia distribución de $Q$. sideroxyla en Monte Grande, y en comparación en zonas bajas que solo se llega a encontrar en sitios con cañadas húmedas. La densidad llega a ser similar (712-1515 ind/ha) mientras que el área basal es menor en las zonas bajas (16.1-30.96 m²/ha) (Díaz-Núñez et al., 2016).

En relación con la altura del arbolado, esta se encontró, en su mayoría, entre 3.60-5 m. Sin embargo, en la asociación bosque de encino $Q$. sideroxyla- $Q$. rugosa se observó una distribución con alturas mayores $(8.57 \pm 1.61$ 
m). En bosques de Quercus la altura depende en gran medida del sitio y las condiciones edáficas y climáticas en las que se desarrollan; las especies de mayor altura se llegan a desarrollar en los bosques mesófilos donde tienen mayor humedad y suelos más ricos y profundos (Park, 2001; Rzedowski, 2006). La diferencia de altura del bosque de encino $Q$. sideroxyla- $Q$. rugosa, comparada con las otras asociaciones, posiblemente se deba a la composición de especies y a su respuesta en diversas condiciones ambientales (Álvarez-Moctezuma et al., 1999; Díaz-Núñez et al., 2012). Este bosque presenta mayor densidad de taxa de hábito arbóreo que llega a alcanzar mayor altura, como es el caso del género Arbutus, y de especies de encino como $Q$. sideroxyla y $Q$. rugosa que en su mayoría llegan a presentar alturas de 8-15 m (Martínez-Calderón et al., 2017), así como su distribución en sitios con suelos con mayor profundidad y en cañadas. En el caso del bosque mixto de Quercus-Pinus, la distribución de alturas, además de estar influenciada por la presencia de taxa de porte bajo y suelos someros, se debe en parte a las diferentes etapas de desarrollo de los pinos con poblaciones de individuos jóvenes. Los valores encontrados $(4.00 \pm 0.23 \mathrm{~m})$ son diferentes a los reportados para poblaciones de Pinus del país, con alturas que oscilan de 8-25 m (Rzedowski, 2006), lo cual se debe posiblemente a las razones mencionadas anteriormente. Sin embargo, los valores de altura del bosque de QuercusPinus son similares a los bosques de Pinus cembroides Zucc. de Zacatecas (4.25 $\pm 3.09 \mathrm{~m}$ ) (Barrera-Zubiaga et al., 2018) que, por su cercanía, pueden tener condiciones ambientes y de sitio muy similares. En este anterior caso se contemplaron especies tanto arbóreas como arbustivas.

Las previas comparaciones sobre la estructura muestran la importancia de llevar a cabo estudios y generar información acerca de los bosques templados. Esto facilitaría realizar comparaciones más amplias respecto a su composición, diversidad y estructura, dada la alta variación climática, estructural y la importancia que presentan para la economía del país. Paradójicamente a su importancia, han sido poco estudiados en comparación a los bosques tropicales y mesófilos de montaña (Martínez-Cruz et al., 2009; Zacarías-Eslava et al., 2011). Así mismo, en el ANPSF hacen falta estudios que permitan comparar zonas y tener ma- yor información de toda el ANP. Los trabajos que existen son principalmente en zonas de 2400-2600 m, cercanas a la comunidad de "La Congoja". Aunque abordan variables estructurales y de diversidad en estudios de distribución y dinámica (Díaz-Núñez et al., 2012; 2016), no se describe la vegetación de la manera que se aborda en este trabajo, dado a que siguieron otros objetivos. Por lo anterior, sería deseable realizar estudios sobre la diversidad y estructura caracterizando tipos de vegetación y asociaciones en diferentes zonas del ANPSF.

\section{Estructura y su relación con el disturbio}

Los disturbios antrópicos tienen influencia directa en la estructura y regeneración de los bosques (Turner et al., 1998; Park, 2001; Rzedowski, 2006). Los disturbios incrementan la densidad de especies pioneras como A. pungens, especie considerada en la mayoría de sus poblaciones, de sucesión secundaria (Siqueiros et al., 2016; Sosa-Ramírez et al., 2016). En el sitio de estudio, esta especie pudo haber sido favorecida por la topografía del lugar, la extracción y producción de carbón en décadas pasadas, así como por incendios de gran magnitud ocurridos en la década de 1950 que causaron pérdidas de bosque de Pinus-Quercus y madroño (CONABIO, 2008). Lo anterior se debe a que $A$. pungens es favorecida por suelos superficiales y la presencia de fuego (Márquez-Linares et al., 2006). Esto se reflejó en las densidades en el bosque de encino $Q$. potosina- $A$. pungens y bosque mixto de Quercus-Pinus, con presencia de $A$. pungens como un componente presente en el estrato arbustivo. Otra especie indicadora de disturbio es $J$. deppeana (Rzedowski, 2006; Díaz-Núñez et al., 2012) que también tiene altos valores de importancia en los bosques estudiados. Al igual que $A$. pungens, pudo haber sido favorecida por el manejo ya que los bosques de Juniperus se desarrollan en sitios con pendientes poco pronunciadas y de fácil acceso. Aunque no fue evaluado en este trabajo, algunas observaciones realizadas muestran que bajo el dosel de J. deppeana, en los bosques de Juniperus, se llegan a establecer plántulas de $Q$. sideroxyla, $Q$. rugosa y A. xalapensis. Esto sugiere una probable tasa de recambio de especies, por lo que se recomendarían realizar futuros estudios para conocer la dinámica de estos bosques. 
La presencia de especies arbóreas de porte bajo, así como los patrones de distribución en las clases diamétricas menores de algunas especies, sugieren que las comunidades estuvieron sujetas a disturbios y están en proceso de recuperación. En bosques templados con mayor tiempo de recuperación, y con ausencia de disturbios, se ha mencionado que los diámetros y las áreas basales que presentan son superiores (Sánchez-Rodríguez et al., 2003; North et al., 2004; Cuevas-Guzmán et al., 2011). Las observaciones de individuos con diámetros grandes, como es el caso de Q. sideroxyla y P. durangensis, así como la distribución diamétrica de especies con mayor valor de importancia en las primeras clases diamétricas (indicando poblaciones juveniles, Fig. 5), sugieren distintas etapas sucesionales, lo cual se debe al aprovechamiento realizado en los bosques estudiados (Sánchez-Rodríguez et al., 2003). Lo anterior es favorable para el objetivo de conservación que tiene actualmente Monte Grande (plan de manejo en revisión), indicando regeneración del bosque. Aunque cabe mencionar que en cañadas de difícil acceso se encuentran bosques de encino de $Q$. sideroxyla- $Q$. rugosa mejor conservados, en términos de composición de especies. Estos bosques presentan poca abundancia de taxones considerados indicadores de disturbios ( $A$. pungens y J. deppeana) y mayor dominancia de taxones considerados de sitios conservados $(Q$. rugosa y Arbutus sp.) (Rzedowski, 2006; Guzmán-Mendoza et al., 2014; Sosa-Ramírez et al., 2016).

En sitios con presencia de especies de pinos como $P$. durangensis, $P$. lumholtzii, $P$. teocote y $P$. strobiformis se observan individuos con clases diamétricas menores. De acuerdo con Bongers et al. (1988), las especies en este patrón presentan una buena reproducción, así como un establecimiento e incorporación natural continuos. Esto es más notorio en $P$. durangensis, donde sus poblaciones están distribuidas en las primeras clases diamétricas (Fig. 5). Lo anterior concuerda con Chapa-Bezanilla et al. (2008), que mencionan el reclutamiento de individuos nuevos y una recuperación moderada en poblaciones de pino, a pesar de las presiones naturales.

En otros bosques de Quercus-Pinus en México, se ha observado que los pinos son más abundantes en las primeras etapas de la regeneración, ya que suelen estar adaptados a la radiación solar directa, a diferencia de los encinos; considerando a los pinos como especies pioneras (González-Espinosa et al., 1991; Almazán-Núñez et al., 2016). Lo anterior discrepa con lo registrado en este estudio. A pesar de que el bosque se encuentra en un estadio temprano de sucesión y los pinos tienen mayor riqueza, difiere con otros bosques debido a la presencia particular de Q. jonesii. Esta es una especie de mayor abundancia, adaptada a suelos superficiales y condiciones de mayor luz; que ha sido citada como pionera y nodriza para otras especies de encinos en bosques templados de San Luís Potosí (Castillo-Lara et al., 2008). Lo anterior indicaría que, en los bosques de Monte Grande, $Q$. jonesii también podría estar actuando como especies nodriza, junto con $A$. pungens, para luego ser una comunidad dominada por pinos.

\section{Implicaciones de manejo}

En la actualidad el manejo del área tiene un enfoque de protección a la biodiversidad y conservación de los servicios ecosistémicos. Las actividades de aprovechamiento maderable del arbolado no se realizan desde aproximadamente hace cuatro décadas (Minnich et al., 1994; Sosa Ramírez et al., 2015), ocasionando la recuperación de los bosques, lo cual es favorable para el objetivo actual de Monte Grande. En el caso de los pinos se sugeriría evitar el corte de estos para que continúen su proceso actual de recuperación, y en los sitios con densidad alta de $A$. pungens reducir su número para facilitar la sucesión ecológica. Considerando la presencia de especies en peligro como Litsea glaucescens Kunth (SEMARNAT, 2010) y de distribución restringida para el estado como Pinus strobiformis (Siqueiros-Delgado et al., 2017), sería deseable continuar con el enfoque actual de conservación y con los apoyos para estos bosques. De esta forma, se puede conservar la presencia de estas especies y continuar con obras de rehabilitación de la propiedad comunal Monte Grande.

Los sitios de altitud más baja en el ANPSF suelen estar sujetos a mayor presión antrópica. Entre las más comunes esta la cacería (legal e ilegal), el aprovechamiento de leña y el pastoreo, además de que el principal problema es la fragmentación del hábitat, por medio de la subdivisión de propiedades y cercado de las mismas (Sosa-Ramírez et al., 2015; 2020). Lo anterior no ocurre en Monte Grande, pues al ser propiedad comunal no se subdivide y solo se tiene 
la cerca perimetral de las 3852 ha. Lo anterior favorece el libre tránsito de la fauna; aunado al enfoque de conservación actual, lo hace un sitio adecuado para la conservación de los recursos naturales de Aguascalientes.

\section{Conclusiones}

Se identificaron 26 especies leñosas presentes en Monte Grande, distribuidas en cinco asociaciones: bosque de $Q$. sideroxyla-Q.rugosa, bosque de $Q$. potosina- $A$. pungens, bosque mixto de Quercus-Pinus, bosque mixto de Juniperus-Quercus y bosque de Juniperus. El bosque de Juniperus es la menos diversa por su alta dominancia de una sola especie; mientras que el bosque mixto de Quercus-Pinus, bosque de $Q$. sideroxyla-Q.rugosa y bosque de $Q$. potosina-A. pungens, presentaron la mayor diversidad. Se encontraron diferencias en la estructura de las asociaciones debido a su composición, condición en la que se desarrollan e historial de manejo de Monte Grande. Existe una alta abundancia de especies que son consideradas pioneras ( $J$. deppeana y A. pungens) y una distribución del diámetro de las especies en las primeras clases diamétricas indicando regeneración.

El presente estudio brinda información de referencia para futuras investigaciones y para considerar en la toma de decisiones en cuanto al manejo del área de estudio. De esta forma se puede buscar conservar los recursos y los beneficios que los bosques templados brindan a la población de las zonas semiáridas y agrícolas que dependen de la captación de agua por parte de los bosques del ANPSF, en los estados de Aguascalientes y Zacatecas.

\section{Contribución de autores}

VMC, JSR y VDN realizaron el trabajo de campo; MSD hizo la confirmación taxonómica de los ejemplares. VMC llevó a cabo el análisis de datos y redactó del artículo. Todos los autores contribuyeron a la discusión, revisión y aprobación del manuscrito final.

\section{Financiamiento}

Esta investigación fue financiada por la Universidad Autónoma de Aguascalientes (UAA) y el Consejo Nacional de Ciencia y Tecnología (CONACyT) a través de la beca de posgrado otorgada al primer autor.

\section{Agradecimientos}

El primer autor agradece al Consejo Nacional de Ciencia y Tecnología (CONACYT), por la beca para realizar sus estudios. Se agradece a Ramón Cuevas Guzmán por sus valiosas enseñanzas en el análisis de datos estructurales, a los compañeros Ricardo García, Juan Daniel Rendón, Luis Alberto Hernández y Alejandro Ruvalcaba por su ayuda en el trabajo de campo; a José Adrián Calzada, Marcelo de Loera y Alejandro Guadalupe Fierros por su ayuda en campo y guía al sitio de estudio. Agradecemos al editor y revisores anónimos por sus contribuciones para el mejoramiento de este manuscrito.

\section{Literatura citada}

Aguirre, O., J. J. Corral, B. Vargas y J. Jiménez. 2008. Evaluación de modelos de diversidad-abundancia del estrato arbóreo en un bosque de niebla. Revista Fitotecnia Mexicana 31(3): 281-289. DOI: https://doi.org/10.35196/rfm.2008.3.281

Alanís-Rodríguez, E., J. Jiménez-Pérez, A. Valdecantos-Dema, M. A González-Tagle, Ó. A. Aguirre-Calderón y E. J. Treviño-Garza. 2012. Composición y diversidad de la regeneración natural en comunidades de Pinus-Quercus sometidas a una alta recurrencia de incendios en el noreste de México. Revista Mexicana de Biodiversidad 83(4): 1208-1214. DOI: https:// doi.org/10.7550/rmb.29708

Almazán-Núñez, R. C., P. Corcuera, L. Parra-Juárez, J. JiménezHernández y G. M. Charre. 2016. Changes in structure and diversity of woody plants in a secondary mixed pine-oak forest in the Sierra Madre del Sur of Mexico. Forests 7(4): 90. DOI: https://doi.org/10.3390/f7040090

Álvarez-Moctezuma, J. G., S. Ochoa-Gaona, B. H. J. De Jong y M. L. Soto-Pinto. 1999. Hábitat y distribución de cinco especies de Quercus (Fagaceae) en la Meseta Central de Chiapas, México. Revista Biología Tropical 47(3): 351-358. DOI: https://doi.org/10.15517/RBT.V4713.19083

Álvarez-Yépiz, J. C., A. Martínez-Yrizar, A. Búrquez y C. Lindquist. 2008. Variation in vegetation structure and soil properties related to land use history of old-growth and secondary tropical dry forests in northwestern México. Forest Ecology Managment 256(3): 355-366. DOI: https://doi. org/10.1016/j.foreco.2008.04.049

Barrera-Zubiaga, E. J. E., D. Granados-Sánchez, R. L. GranadosVictorino y M. Luna-Cavazos. 2018. Characterization 
of four pinyon pine forests in the state of Zacatecas, Mexico. Revista Chapingo Serie Ciencias Forestales y del Ambiente 24(3): 275-296. DOI: https://doi.org/10.5154/r. rchscfa.2017.03.018

Bongers, F., J. Popma, J. Meave y J. Carabias. 1988. Structure and floristic composition of the lowland rain forest of LoS Tuxtlas, México. Vegetation 74: 55-80. DOI: https://doi. org/10.1007/BF00045614

Calderón-Aguilera, L., V. Rivera-Monroy, L. Porter-Bolland, A. Martínez-Yrízar, L. Ladah, M. Martínez-Ramos, J. Alcocer, A. Santiago-Pérez, H. Hernández-Aranda, V. Reyes-Gómez, D. Pérez-Salicrup, V. Díaz-Nuñez, J. Sosa-Ramírez, J. HerreraSilveira y A. Búrquez. 2012. An assessment of natural and human disturbance effects on Mexican ecosystems: current trends and research gaps. Biodiversity and Conservation 21: 589-617. DOI: https://doi.org/10.1007/ s10531-011-0218-6

Castillo-Lara, P., J. A. Flores-Cano, J. R. Aguirre-Rivera y R. Yeaton. 2008. Dinámica sucesional del encinar de la sierra de Álvarez, San Luis Potosí, México. Madera y Bosques 14(1): 21-35. DOI: https://doi.org/10.21829/ myb.2008.1411215

Challenger, A. 2003. Conceptos generales acerca de los ecosistemas templados de montaña de México y su estado de conservación. In: Sánchez, Ó., E. Vega, E. Peters y O. Monroy-Vilchis (eds.). Conservación de ecosistemas templados de montaña en México. Instituto Nacional de Ecología (INE), Secretaría del medio ambiente y recursos naturales (SEMARNAT). Cd. Mx., México. 316 pp.

Challenger, A. y J. Soberón. 2008. Los ecosistemas terrestres de México. In: Capital natural de México, Vol. I. Conocimiento actual de la biodiversidad. Comisión Nacional para el Conocimiento de la Biodiversidad (CONABIO) Cd. Mx., México. pp. 87-108.

Chapa-Bezanilla, D., J. Sosa-Ramírez y A. de Alba-Ávila. 2008. Estudio multitemporal de fragmentación de los bosques en la Sierra Fría, Aguascalientes, México. Madera y Bosques 14(1): 37-51. DOI: https://doi.org/10.21829/ myb.2008.1411216

Chazdon, R. L. 2003. Tropical forest recovery: legacies of human impact and natural disturbances. Perspectives in Plant Ecology Evolution and Systematics 6(1-2): 51-71. DOI: https://doi.org/10.1078/1433-8319-00042
Colwell, R. K. 2016. EstimateS version 9.1.0: Statistical estimation of species richness and shared species from samples. http://viceroy.eeb.uconn.edu/Estimates/ (consultado abril de 2019).

Colwell, R. K., A. Chao, N. J. Gotelli, S. Y. Lin, C. X. Mao, R. L. Chazdon y J. T. Longino. 2012. Models and estimators linking individual-based and sample-based rarefaction, extrapolation, and comparison of assemblages. Journal of Plant Ecology 5(1): 3-21. DOI: https://doi.org/10.1093/jpe/ rtr044

CONABIO. 2008. La Biodiversidad en Aguascalientes: Estudio de estado. Comisión Nacional para el Conocimiento y Uso de la Biodiversidad (CONABIO), Instituto de Medio Ambiente del Estado de Aguascalientes (IMAE), Universidad Autónoma de Aguascalientes (UAA). Cd. Mx., México. 385 pp.

CONAFOR. 2014. Inventario estatal forestal y de suelos, Aguascalientes. Comisión Nacional Forestal. Cd. Mx., México. 136 pp.

COTAS. 2006. Escenarios del agua 2015 y 2030 en el acuífero interestatal de Ojocaliente-Aguascalientes-Encarnación: acciones para un desarrollo con sostenibilidad ambiental. Comité Técnico de Aguas Subterráneas del acuífero interestatal de Ojocaliente-Aguascalientes-Encarnación, A.C., Informe final. México, D.F. México.

Cuevas-Guzmán, R., E. A. Cisneros-Lepe, E. J. Jardel-Peláez, E. V. Sánchez-Rodríguez, L. Guzmán-Hernández, N. M. NúñezLópez y C. Rodríguez-Guerrero. 2011. Análisis estructural y de diversidad en los bosques de Abies de Jalisco, México. Revista Mexicana de Biodiversidad 82(4): 1219-1233. DOI: http://dx.doi.org/10.22201/ib.20078706e.2011.4.741

Daget, P. y M. Godron. 1982. Analyse de l'ecologie des espéces dans les communautés. Ecologia Mediterranea 8(4): 206.

De la Cerda, L. M. 1999. Encinos de Aguascalientes. 2a ed. Universidad Autónoma de Aguascalientes. Aguascalientes, México. $88 \mathrm{pp}$.

Del Río, M., F. Montes, I. Cañellas y G. Montero. 2003. Revisión: Índices de diversidad estructural en masas forestales. Investigaciones Agrarias: Sistemas de Recursos Forestales 12(1): 159-176. DOI: https://doi.org/10.5424/795

Díaz-Núñez, V., J. Sosa-Ramírez y D. R. Pérez-Salicrup. 2012. Distribución y abundancia de las especies arbóreas y arbustivas en la Sierra Fría, Aguascalientes, México. Polibotánica 34: 99-126. 
Díaz-Núñez, V., J. Sosa-Ramírez y D. R. Pérez-Salicrup. 2016. Vegetation patch dynamics and tree diversity in a conifer and oak forest in central Mexico. Botanical Sciences 94(2): 229-240. DOI: https://doi.org/10.17129/botsci.284

Di-Rienzo, J. A., F. Casanoves, M. G. Balzarini, L. Gonzalez, M. Tablada y C. W. Robledo. 2016. InfoStat versión 2016. Grupo InfoStat, FCA, Universidad Nacional de Córdoba. Córdoba, Argentina. http://www.infostat.com.ar (consultado julio de 2019).

Domínguez-Gómez, T. G., B. N. Hernández González, H. González Rodríguez, I. Cantú Silva, E. Alanís Rodríguez y M. S. Alvarado. 2018. Estructura y composición de la vegetación en cuatro sitios de la Sierra Madre Occidental. Revista Mexicana de Ciencias Forestales 9(2): 10-34. DOI: https:// doi.org/10.29298/rmcf.v9i50.227

González, V. L. 1986. Contribución al conocimiento del género Quercus (Fagaceae) en el Estado de Jalisco. Universidad de Guadalajara. Jalisco, México. 240 pp.

González-Espinosa, M., P. Quintana-Ascencio, N. RamírezMarcial y P. Gaytán-Guzmán. 1991. Secondary succession in disturbed Pinus-Quercus forests in the highlands of Chiapas, Mexico. Journal of Vegetation Science 2(3): 351-350. DOI: https://doi.org/10.2307/3235927

Guzmán-Mendoza, R., J. A. Zavala-Hurtado, G. Castaño-Meneses y J. L. León-Cortés. 2014. Comparación de la mirmecofauna en un gradiente de reforestación en bosques templados del centro occidente de México. Madera y Bosques 20(1): 7183. DOI: https://doi.org/10.21829/myb.2014.201177

INEGI. 2000. Conjunto de datos vectoriales. Climas (Unidades climáticas). Continuo Nacional. Escala 1:1,000,000. Instituto Nacional de Estadística y Geografía. Aguascalientes, México. Jost, L. 2006. Entropy and diversity. Oikos 113(2): 363-375. DOI: https://doi.org/10.1111/j.2006.0030-1299.14714.x

Kappelle, M. y J. G. Van Uffelen. 2006. Altitudinal zonation of montane oak forests along climate and soil gradients in Costa Rica. In: Kappelle, M. (ed.). Ecology and Conservation of Neotropical Montane Oak Forests. Springer-Verlag. Berlin, Germany. pp. 39-50.

López-Hernández, J. A., O. A. Aguirre-Calderón, E. AlanísRodríguez, J. C. Monárrez-González, M. A. González-Tagle y J. Jiménez-Pérez. 2017. Composición y diversidad de especies forestales en bosques templados de Puebla, México. Madera y Bosques 23(1): 39-51. DOI: https://doi. org/10.21829/myb.2017.2311518
Maldonado-Sánchez, E. A. y F. Maldonado-Mares. 2010. Estructura y diversidad arbórea de una selva alta perennifolia en Tacotalpa, Tabasco, México. Universidad y Ciencia 26: 235245.

Márquez-Linares, M. A., S. González Elizondo y R. Alvarez-Zagoya. 1999. Componentes de la diversidad arbórea en bosques de pino encino de Durango, Méx. Madera y Bosques 5(2): 6777. DOI: https://doi.org/10.21829/myb.1999.521348

Márquez-Linares, M. A., E. Jurado y S. González. 2006. Algunos aspectos de la biología de la manzanita (Arctostaphylos pungens HBK.) y su papel en el desplazamiento de bosques templados por chaparrales. Ciencia 9: 57-64.

Martín-Clemente, C. R., J. Sosa-Ramírez, M. Maass-Moreno, J. J. Luna-Ruíz, A. J. Meráz-Jiménez y E. Flores-Ancira. 2015. Comparación de métodos para estimar disponibilidad hídrica en cuencas forestales. Terra latinoamericana 33: 17-26.

Martínez-Calderón, V. M., M. E. Siqueiros-Delgado y J. MartínezRamírez. 2017. Checklist of the genus Quercus (Fagaceae) of Aguascalientes, México. Check List 13(1): 2045. DOI: https://doi.org/10.15560/13.1.2045

Martínez-Cruz, J., O. Téllez-Valdés y G. Ibarra-Manríquez. 2009. Estructura de los encinares de la sierra de Santa Rosa, Guanajuato, México. Revista Mexicana de Biodiversidad 80(1): 145-156. DOI: http://dx.doi.org/10.22201/ ib.20078706e.2009.001.592

McCune, B. y M. J. Mefford. 2011. PC-ORD Multivariate analysis of ecoclogical data version 6.0. MjM Software. Gleneden Beach, EUA.

Minnich, R. A., J. Sosa Ramírez, V. E. Franco, W. J. Barry y M. E. Siqueiros. 1994. Reconocimiento preliminar de la vegetación y de los impactos de las actividades humanas en la Sierra Fría, Aguascalientes, México. Investigación y Ciencia 12: 23-29.

Moreno, C. E., F. Barragán, E. Pineda y N. P. Pavón. 2011. Reanalizando la diversidad alfa: alternativas para interpretar y comparar información sobre comunidades ecológicas. Revista Mexicana de Biodiversidad 82(4): 1249-1261. DOI: http://dx.doi.org/10.22201/ib.20078706e.2011.4.745

Mueller-Dombois, D. y H. Ellenberg. 1974. Aims and methods of vegetation ecology. Wiley. New York, EUA. 547 pp.

Návar-Cháidez, J. J. y S. González-Elizondo. 2009. Diversidad, estructura y productividad de bosques templados de Durango, México. Polibotánica 27: 71-87. 
North, M., J. Chen, B. Oakley, B. Song, M. Rudnicki, A. Gray y J. Innes. 2004. Forest Stand Structure and Pattern of OldGrowth Western Hemlock/Douglas-Fir and Mixed-Conifer Forests. Forest Science 50(3): 299-311.

Olvera-Vargas, M. B. y L. Figueroa-Rangel. 2012. Caracterización estructural de bosques montanos dominados por encino en el centro-occidente de México. Ecosistemas 21(1-2): 74-84. DOI: https://doi.org/10.7818/ECOS.28

Park, A. D. 2001. Environmental influences on post-harvest natural regeneration in Mexican pine-oak forest. Forest Ecology and Management 144: 213-223. DOI: https://doi. org/10.1016/S0378-1127(00)00372-8

Rosete-Vergés, F. A., J. L. Pérez-Damián, M. Villalobos-Delgado, E. N. Navarro-Salas, E. Salinas-Chávez y R. Remond-Noa. 2014. El avance de la deforestación en México 1976-2007. Madera y Bosques 20(1): 21-35. DOI: https://doi.org/10.21829/ myb.2014.201173

Rzedowski, J. 2006. Vegetación de México. Comisión Nacional para el Conocimiento y Uso de la Biodiversidad-libro electrónico. México, D.F., México. https://www.biodiversidad.gob. $\mathrm{mx} /$ publicaciones/librosDig/pdf/VegetacionMx_Cont.pdf (consultado abril de 2019).

Sáenz-Romero, C., E. Snively y R. Lindig-Cisneros. 2003. Conservation and restoration of pine forest genetic resources in Mexico. Silvae Genetics 52: 233-237.

Sánchez, Ó., E. Vega, E. Peters y O. Monroy-Vilchis. 2003. Conservación de ecosistemas templados de montaña en México. Instituto Nacional de Ecología (INE) Secretaría del Medio Ambiente y Recursos Naturales (SEMARNAT). Cd. Mx., México. 316 pp.

Sánchez-Rodríguez, V. E., L. López-Mata, E. García-Moya y R. Cuevas-Guzmán. 2003. Estructura, composición florística y diversidad de especies leñosas de un bosque mesófilo de montaña en la Sierra de Manantlán, Jalisco. Boletín de la Sociedad Botánica de México 73: 17-34. DOI: http://dx.doi. org/10.17129/botsci.1676

SEMARNAT. 2010. NORMA Oficial Mexicana NOM-059SEMARNAT-2010. Protección ambiental-Especies nativas de México de flora y fauna silvestres-Categorías de riesgo y especificaciones para su inclusión, exclusión o cambio-Lista de especies en riesgo. Secretaría del Medio Ambiente y Recursos Naturales. Diario Oficial de la Federación. Cd. Mx., México. http://dof.gob.mx/ nota_detalle.php?codigo $=5173091 \&$ fecha $=30 / 12 / 2010$ (consultado abril de 2021).

Siqueiros, M. E. 1999. Coníferas del Estado de Aguascalientes. Universidad Autónoma de Aguascalientes. Aguascalientes, México. 67 pp.

Siqueiros, M. E., J. A. Rodríguez-Avalos, J. Martínez-Ramírez y J. C. Sierra-Muñoz. 2016. Situación actual de la vegetación del estado de Aguascalientes, México. Botanical Sciences 94(3): 455-470. DOI: http://dx.doi.org/10.17129/ botsci.466

Siqueiros-Delgado, M. E., R. S. Miguel, J. A. Rodríguez-Avalos, J., Martínez-Ramírez y J. C. Sierra-Muñoz. 2017. Richness and Current Status of Gymnosperm Communities in Aguascalientes, Mexico. Aliso: A Journal of Systematic and Evolutionary Botany 35(2): 97-105. DOI: http://dx.doi. org/10.5642/aliso.20173502.06

Sosa-Ramírez, J., A. Breceda-Solís, C. L. Jiménez-Sierra, L. I. Iñiguez-Dávalos y A. Ortega-Rubio. 2015. Capitulo XIX. Los ecosistemas de la Sierra Fría en Aguascalientes y su conservación. In: Ortega-Rubio, A., M. J. PinkusRendón e I. C. Espitia- Moreno (eds.). Las Áreas Naturales Protegidas y la Investigación Científica en México. Centro de Investigaciones Biológicas del Noroeste, S.C., Universidad Autónoma de Yucatán y Universidad Michoacana de San Nicolás de Hidalgo. La Paz, Mérida y Morelia, México. Pp. 447-472. https://cibnor.repositorioinstitucional.mx/jspui/ bitstream/1001/1129/1/PUB-CAPITULOS-LIBROS-980.PDF (consultado abril de 2021).

Sosa-Ramírez, J., O. Moreno-Rico, G. Sánchez-Martínez, J. J. Luna-Ruiz y M. E. Siqueiros-Delgado. 2016. Contribución al conocimiento ecológico del chaparral de manzanita (Arctostaphylos pungens Kunth) en la Sierra Fría, Aguascalientes. Universidad Autónoma de Aguascalientes. Aguascalientes, México. 105 pp.

Sosa-Ramírez, J., V. Díaz-Núñez, D. R. Pérez-Salicrup, F. Tafoya y L. Porter-Bolland. 2020. Interacción sociedad-paisajes: elementos para el manejo de ecosistemas en la Sierra Fría, Aguascalientes, México. Agricultura, Sociedad y Desarrollo 17: 415-433.

Turner, M. G., W. L. Baker, C. J. Peterson y R. K. Peer. 1998. Factors influencing succession: Lessons from large, infrequent natural disturbances. Ecosystems 1: 511-523. DOI: https:// doi.org/10.1007/s100219900047 
Zacarías-Eslava, L. E., G. Cortejo-Tenorio, J. Cortés-Flores, N. González-Castañeda y G. Ibarra-Manríquez. 2011. Composición, estructura y diversidad del cerro El Águila, Michoacán, México. Revista Mexicana de Biodiversidad 82(3): 854-869. DOI: http://dx.doi.org/10.22201/ ib.20078706e.2011.3.684 